DIGITAL COMMONS
@ UNIVERSITY OF SOUTH FLORIDA

Journal of African Conflicts and

Peace Studies

Volume 4 | Issue 2

Article 5

January 2021

\title{
Migrations internationales. Entre enjeux traditionnel et nouveaux défis
}

Leonidas Ndayisaba

University of Burundi, leonidasndayisab@hotmail.com

Follow this and additional works at: https://digitalcommons.usf.edu/jacaps

Part of the International Relations Commons

\section{Recommended Citation}

Ndayisaba, Leonidas (2021) "Migrations internationales. Entre enjeux traditionnel et nouveaux défis," Journal of African Conflicts and Peace Studies: Vol. 4: Iss. 2, .

DOI: https://doi.org/10.5038/2325-484X.4.2.1150

Available at: https://digitalcommons.usf.edu/jacaps/vol4/iss2/5

This Article is brought to you for free and open access by the Open Access Journals at Digital Commons @ University of South Florida. It has been accepted for inclusion in Journal of African Conflicts and Peace Studies by an authorized editor of Digital Commons @ University of South Florida. For more information, please contact digitalcommons@usf.edu. 


\section{Migrations internationales. Entre enjeux traditionnel et nouveaux défis}

\section{Cover Page Footnote}

Migrations humaines; in http://www.wikipedia.com visité le 28 décembre 2015. Jaffrelot Christophe \& Lequesne Christian (dir.) (2009) : L'enjeu mondial. Les migrations, Paris : Presses des Sciences PôL'Express, pp. 11-14. HCR, Tendances mondiales, premier semestre 2015 et 2016, Genève; UNHCR: Une hausse sans précédent des déplacements forcés pourrait marquer l'année 2015 in http://www.unhcr.fr visité le 19 décembre 2015 KNOMAD (Partenariat mondial pour les connaissances sur le développement et les migrations), Recueil des statistiques sur les migrations et les envois de fonds ; in http://wwwworldbank.org visité le 14 octobre 2016. KNOMAD, ibidem. Kirsch Olivier, op. cit., pp. 12-14. Collins Alain (2013): Contemporary Security studies, Oxford: Oxford University Press, pp.138-139. KNOMAD, op. cit. Banque Mondiale (2019) : Rapport de la Banque Mondiale. Leveraging Economic migration for development. A briefing for the World Bank Board, Washington : World Bank Group ou http://www.knomad.org, visité le 10.01.2020. Voir également Banque Mondiale (2018) : Groundswell : Preparing or internal climate Migration, Washington DC : World Bank Group. http://www.akuf.de visité le 11.01.2020. www.fr.wikipedia.com visitée le 15.12.2019. De Montclos M. A.P « Les flux de réfugiés : un enjeu politique au Sud comme au Nord », La Chronique du CEPED, Octobre-Décembre 1998, №31, p. 1. Presque à la création de l'Organisation de l'Unité Africaine (OUA) en 1964, voir Scandinavian Institute of Africa Studies, Refugee problem in Africa, Uppsala, 1967, pp.14-15. Global Trends, Forced Displacement en 2015, http://www.unhcr.org consulté le 15 novembre 2016. Article 1, alinéa 2 de la Convention de l'OUA régissant les aspects propres aux problèmes des réfugiés en Afrique de 1969. Drain Michel \& Dubernet Cécile (2018) : Relations internationales, 23ème édition, Bruxelles : Ed. Bruylant, pp.86-87. Rapport HCR 2018 ; voir également le site de la Deutsche Welle ; www.dw.com. Voir Rapport pour le PNUE en 1985. Le Tsunami de 2003. Swain Aschok,"Environmental migration and conflict dynamics: Focus on developing regions", Third World Quarterly, Vol. 17, N5, 1996, pp.959-973; voir également HCR, op. cit., p.2. Lemaitre Th., "Réfugiés environnementaux, de quoi parle-t-on? », http://www.vuessurlemonde.com visité le 15 novembre 2015. Collins Alan : Op. Cit., p. 138. Article 1. http://www.histoire-immigration.fr visité le 10 janvier 2016. Kirsch Olivier, op. cit., p. 20. De Legge, "Le Costa Rica, le refuge des retraités américains", in http://www.journaldunet.com visité le 12 novembre 2016. Convention relative aux statuts des apatrides du 28 septembre 1954, article 1, alinéa 1. UNHCR, UNHCR Global trends Displacement the new 21 st century challenge, Genève, 2012, p.1. Propos d'Antonio Guterres, HCR, op.cit., pp. 2-3. Echec jusqu'aujourd'hui de la politique des quotas en Europe, la question de l'immigration s'invite dans la campagne américaine, tri de réfugiés sur la frontière avec la Hongrie alors qu'en réalité la Turquie (1,8 millions) et le Liban (209 réfugiés pour 1000 habitants) accueillent le gros des réfugiés syriens pour la plupart. Propos des responsables des camps, jeudi 04 novembre 2015. HCR, op. cit, p. 2. Collins Allan : Op. Cit., pp. 138-139. Bendl Regine / Hanappi-Egger Edeltraud \& Hofmann Roswitha (2012) : Diversität und Diversitätsmanagement, Wien : Facultas Verlag AG ; Garner-Moyer Hélène (2006) : « Gestion de la diversité et enjeux de la gestion des ressources humaines »; in Management et Avenir, 2006/1 ( $\left.\mathrm{N}^{\circ} 7\right)$, pp. 23-42. Rolland Cécilia (2013) : Decreased Polarization through economic integration ? A study of ethnic polarization of members of the Rwandese diaspora 19 years after the genocide, Master thesis in Peace and conflict studies, Uppsala University : DPCR. National Intelligence Council (2008) : « Democratization in Africa : What Progress toward Institutionalization? » Conference Report, Februar ; Teshome Wondwosen (2009) : "Opposition Parties and the Politics of Opposition in Africa : A critical Analysis »; in International Journal of Human and Social Sciences, $4: 5$, pp. 287-301. Barret Francois (1952): Histoire du travail, Col. Que sais-je ? N 164, Paris : PUF, pp.51-58 ; Convention N50 du 20 juin 1936 relative au recrutement des travailleurs indigènes approuvée par la loi du 10 septembre 1947, BO 1948, p. 1023 ; Ministère des Colonies (1932) : Rapport sur l'Administration belge du Ruanda-Urundi, p. 98. Rapport sur l'Administration belge du Ruanda-Urundi, 1959. Chrétien J-Pierre : « La crise écologique de l'Afrique orientale au début du XXème siècle. Cas de l'Imbo au Burundi entre 1890 et 1916 ", in Université du 
Burundi (1985) : Questions sur la paysannerie au Burundi, Bujumbura : FLSH, pp. 55-56. Voir à ce sujet Gahama Joseph, «La question des réfugiés », in Chrétien J-Pierre \& Mukuri Melchior (sous la dir.) (2002), Burundi, la fracture identitaire. Logiques de violence et certitudes «ethniques», Paris, Karthala, pp.225-238. Voir Boddaert Mathilde, «Réfugiés burundais », http://www.crisisgroup.org visité le 14 novembre 2016 ; le site www.arib.info visité le 14.01.2020. ONPRA, HCR \& IGEBU, Rapport du HCR au 31 octobre 2016. Burundi Eco du 01 juin 2018. Magazine Iwacu, N¹, Novembre 2011 : « Où sont nos cerveaux ? ». 18 professeurs ont presté dans ce pays dont 5 au Kigali Institute of Education (KIE); Voir Midende Gilbert, Problématique de la fuite des cerveaux à l'université du Burundi, Bujumbura, PNUD, 2007. Iwacu N 1 de Novembre 2011: Interview de Déo Christian Haringanji : « Pourquoi partent-ils, comment peuvent-ils revenir? pp.6-8 et celle du Prof. Julien Nimubona : « Il faut valoriser la science », p. 29-30. Voir à ce sujet : "Stateless in Burundi : Omanis search for a nationality" ; in http://www.english.alarabiya.net consulté le 04 juillet 2017. Ntwari Bernard (2013) : Risque d'apatridie au Burundi: Cas des Omanais vivant au Burundi, Mémoire, DESS en Droits de l'Homme et Résolution pacifique des conflits, p. 9. Ajoutons qu'on peut trouver dans une seule et même famille à la fois des membres apatrides et d'autres ayant la nationalité omanaise, passeport, séjour alternatif dans les deux pays, etc. Voir également "Statelessness in Burundi »; in: http://www.iwacu-burundi.org/english consulté le 04 juillet 2017. Voir Le Monde du 11 mai 2017 et www.rfi.fr consulté le 10 mai 2017. Pour de plus amples informations sur l'enjeu de l'apatridie et les solutions envisageables, consulter UNHCR \& UIP (2005) : Nationalité et Apatridie. Un guide pour les parlementaires, Genève Uvin Peter (1998) : Aiding violence. The development enterprise in Rwanda, Connecticut: Kumarian Press, p. 34; voir Willame J-Claude (1995): Aux sources de l'hécatombe rwandaise, Bruxelles, Cedaf. Selon le gouvernement burundais, les attaques de Kabarore en juillet 2015 et la récente attaque du 17 novembre 2019 contre une position de l'armée régulière ont été menées par de groupes rebelles venus du Rwanda où se trouvent une importante communauté de réfugiés burundais depuis 2015, et ces deux évènements font l'objet de tensions diplomatiques entre les deux pays. Attribution d'une médaille à l'effigie de Nansen et d'une enveloppe de 100.000\$US. Fombeno Tagum H.J., op. cit, p.250. Rapport HCR 2010 tenant compte de ce mouvement consécutif à l'application de l'Accord d'Arusha. D'autres vont s'y ajouter à partir de 2015, nous y reviendrons plus tard. République du Burundi, Politique nationale de la diaspora, Bujumbura, MREC, 2015, pp. 12-15. Voir Wihtol de Wenden Cathérine : Les nouvelles migrations. Lieux, hommes, politiques, Paris, Ellipses (tiré de http://www.cairn.info visité le 03 .01.2016). Manirakiza Egide, op. cit, pp.15-16. Reconduit tous les cinq ans par une Résolution de l'Assemblée Générale des Nations Unies, le mandat du HCR est devenu illimité depuis 2004 «jusqu'à ce que la question des réfugiés dans le monde soit définitivement résolue». 


\section{Introduction}

Depuis le début de la guerre civile ou «printemps arabe » dans les pays du Maghreb et au Moyen-Orient en 2011, l'attention internationale en 2015 portait particulièrement sur l'escalade du conflit interne syrien entre les insurgés d'un côté et les troupes régulières du pouvoir de Damas de l'autre, faisant oublier, pour un moment, les conséquences de cette guerre fratricide. Il faudra attendre le deuxième trimestre de l'année 2015 pour que l'attention internationale découvre une autre dimension de cette guerre, le drame humain qui l'accompagne en termes de destructions humaines et matérielles, à savoir un pays à reconstruire entièrement, les déplacements forcés de populations tant à l'intérieur qu'à l'extérieur de la Syrie composés de flux de réfugiés syriens, mais aussi irakiens, érythréens qui, à leur péril, traversent la Mer Méditerranée en vue de demander asile, sinon trouver refuge sans doute à jamais, dans les pays européens et nord-américains.

Face à ce drame humain, la réaction des pays européens et occidentaux en général est, pour un tant soit peu, de reconnaître le drame personnel et humain en face et d'accueillir ces migrants forcés, prenant le contre-pied des politiques d'immigration jusqu'alors restrictives pratiquées dans beaucoup de pays ${ }^{1}$. Il s'agit de flux d'êtres humains qui accompagnent les conflits internes ou interétatiques dans le monde, particulièrement après la fin de la guerre froide pour bien des pays ou régions dits du Tiers monde, en Amérique Latine, en Afrique et en Asie où se déroulent la plupart de ces conflits intra-étatiques.

Le rapport annuel de 2019 du Haut-Commissariat des Nations Unies pour les réfugiés (HCR) fait état de 70,8 millions de personnes ayant fui la guerre, les persécutions ou les conflits, un niveau de déplacements forcés que ladite organisation n'avait jamais atteint pendant les soixante et dix années de son existence. Ce ne sont que des tendances actuelles dans la mesure où elles ne reflètent pas réellement toute la situation actuelle de la crise au Venezuela dont 4 millions de ressortissants seraient dispersés à travers l'Amérique latine principalement et ailleurs dans le monde et d'autres groupes non enregistrés à travers le monde; bref, cet ensemble constituerait la $21^{\text {ème }}$ nation au monde ${ }^{2}$. La question du flux des réfugiés revêt une importance cruciale tant en amont, dans leurs pays d'origine qui perdent subitement, parfois à jamais, des milliers de citoyens, qu'en aval, dans les pays d'accueil, qui hébergent des milliers d'étrangers à intégrer, former et enfin ouvrir de nouvelles opportunités ${ }^{3}$.

L'objectif de cette contribution est, au-delà de l'examen de la question des migrations internationales sur les plans politique, juridique et social, d'analyser les défis et les enjeux que ces nouveaux flux d'êtres humains posent pour leurs pays d'origine et ceux d'accueil. Quelle est la nature des nouveaux flux migratoires? Quels sont les défis et les enjeux du traitement de cette question tant pour les pays d'origine que les pays d'accueil? Autant de questions auxquelles je me propose de répondre dans cet article.

\footnotetext{
${ }^{1} 1.000 .000$ de réfugiés pour la plupart Syriens (Irakiens, Érythréens, Afghans) en Allemagne, 37.501 en Autriche, 10.000 en France.

${ }^{2}$ Rapport UNHCR (2019): Global Trends: Forced displacement in 2018, Genève.

${ }^{3}$ Pour l'année 2016, l'Allemagne (Gouvernement fédéral et Etats fédérés) devait consacrer 19 milliards d'Euros pour l'intégration des nouveaux réfugiés.
} 
L'idée de traiter ces questions vient d'une réflexion sous forme de présentation partagée en octobre 2015 lors d'un séminaire organisé conjointement par l'Université d'Osaka (Japon) et le «Dag Hammaskjörg Institute of Peace Studies» du «Copperbelt University» de Kitwe (Zambie) sur l'enjeu de la question des réfugiés dans le monde. La question des réfugiés ne constitue en réalité qu'un sous-thème dans la galaxie des déplacements forcés à travers le monde. Nous y reviendrons en détails plus tard. Ainsi me vient-il l'idée d'aller au-delà de la question des réfugiés, mais plutôt de traiter l'ensemble des migrations internationales avec des illustrations sur les pays de la Région des Grands Lacs africains en général et le Burundi en particulier.

Le présent article est le fruit d'un travail réalisé à partir de la lecture des rapports, de la littérature grise, des revues et des entretiens semi-directifs conduits auprès des réfugiés, anciens ou actuels, et des personnes et institutions qui, dans leur vie professionnelle, s'occupent des migrations dans différents pays tels que le Haut-Commissariat des Nations Unies pour les Réfugiés (HCR), l'Office Burundais pour la Protection des Apatrides (ONPRA) qui, travaillant en synergie au Burundi, disposent de statistiques détaillées et actualisées. D'autres sources procèdent d'un recoupement d'informations recueillies auprès de différents services d'immigration, d'émigration, les qualifications des migrants et les envois de fonds pour estimer le nombre de migrants dans le monde. Ce travail a été réalisé en 2016 par le Partenariat mondial pour les connaissances sur le développement et les migrations (KNOMAD) de la Banque Mondiale dans son Recueil des Statistiques sur les migrations et les envois de fonds pour 214 pays et territoires conduisant également à recenser les migrants dans le monde, lesquels ont envoyé cette année 601 milliards de dollars à leurs familles d'origine. Ce rapport qui est publié chaque année depuis 2007 a fait l'objet particulier de commentaires dans la presse internationale.

\section{Migrations internationales: toujours une réalité au XXI ${ }^{\text {me }}$ siècle}

\section{Un phénomène historique}

Les migrations humaines à savoir des déplacements d'un individu ou groupes d'individus de leur cadre habituel de vie sont aussi vielles que l'humanité : de simples déplacements de populations au gré d'une économie de subsistance et le nomadisme constituaient certes des mouvements d'individus à des dizaines et centaines de kilomètres à la recherche de ressources naturelles, mais ils ne peuvent pas pour autant être considérés comme des mouvements migratoires. Dans cet ordre d'idées, les migrations préhistoriques de petits groupes sur de courtes distances ont conduit, à travers des millénaires, au peuplement de l'Asie, de l'Australie et de l'Amérique avant la constitution d'actuels ensembles étatiques élargis ${ }^{4}$. D'autres formes telles que de lointains pèlerinages, les voyages de commerçants sur les routes de la soie, les invasions celtes, les conquêtes musulmanes, répondaient plutôt à des objectifs politiques propres aux élites dirigeantes.

Ces déplacements diffèrent des migrations actuelles dites postindustrielles qui conduisent plutôt à un changement définitif de résidence, de cadre de vie. D'après Christophe Jaffrelot et Christian Lequesne, le sens de la migration se définit par la mobilité collective, volontaire ou forcée des

${ }^{4}$ Migrations humaines; in http://www.wikipedia.com visité le 28 décembre 2015. 
hommes sur de grandes distances: la fondation de cités grecques sur le pourtour de la Méditerranée dès le VIII ${ }^{\text {ème }}$ siècle av. J.-C., la formation d'une diaspora juive dès le IV ${ }^{\text {ème }}$ siècle avant notre ère sont, entre autres, des exemples de migrations massives. Ce mouvement s'amplifie à partir du XIX ${ }^{\text {ème }}$ siècle avec la modernisation des sociétés européennes perceptible à travers la révolution industrielle, la formation de grandes entités politiques et économiques, le développement des moyens de transports et communications permettant à des millions de personnes de se déplacer à travers les continents. Selon le même auteur, de ces déplacements entre 1850 et 1914, il en résulte qu'un million d'Européens s'embarquent en moyenne à destination des Amériques, soit une cinquantaine de millions d'hommes et de femmes en moins d'un siècle, de 1820 à 1930, à la veille du XX ${ }^{\text {ème }}$ siècle.

Le phénomène migratoire du vingtième siècle résulte de plusieurs facteurs d'ordre politique, économique, socio-culturel, technologique, géographique et autres dont les statistiques suivantes font état de l'ampleur de ces déplacements humains. De 75 millions de migrants internationaux en 1965, ils atteignent 191 millions en 2005, puis 250 millions en 2015 au point que pour certains pays, comme les Îles du Cap Vert, la communauté vivant à l'étranger est de loin supérieure à la population du pays d'origine ${ }^{5}$. Il s'agit des migrations internationales parmi lesquelles il faut distinguer des migrations forcées («illegal, undocumented migration » dit-on aussi) telles que les flux de réfugiés. Ainsi, si l'année 2015 a même été une année record en termes de flux de réfugiés, il faudra remonter à 1992 pour atteindre le nombre actuel et l'ensemble des déplacés forcés atteint 60 millions pour la première fois (65 million en 2016, soit $1 \%$ de la population mondiale) ; bref un être humain sur 12 est aujourd'hui une personne qui a été forcée de quitter son foyer6. Selon l'édition 2016 du Recueil des statistiques sur les migrations et les envois de fonds publiés par le Partenariat mondial pour les connaissances sur le développement et les migrations de la Banque mondiale, le nombre de migrants internationaux devrait dépasser actuellement le chiffre record de 250 millions de personnes.

Cette situation s'avère paradoxale dans la mesure où quand les populations concernées cherchent de meilleures opportunités économiques, les pays en développement qui connaissent une croissance rapide continuent pourtant d'attirer un grand nombre de populations d'autres régions du monde en développement ${ }^{7}$. Dans les pays du Golfe persique par exemple avec une économie émergente et diversifiée, le nombre de travailleurs immigrés dépassent de loin celui des nationaux.

Ces données chiffrées, quoique différentes en fonction des sources et des méthodes utilisées, attestent néanmoins des développements des dernières décennies en termes de migration. Le premier développement consiste dans les raisons des migrations qui se sont multipliées et par là compliquées: à la pauvreté structurelle des pays du Tiers-Monde ou des circonstances conjoncturelles consécutives aux guerres, à la recherche de l'emploi et une amélioration des

\footnotetext{
5 Jaffrelot Christophe \& Lequesne Christian (dir.) (2009) : L'enjeu mondial. Les migrations, Paris : Presses des Sciences Pô-L'Express, pp. 11-14.

${ }^{6}$ HCR, Tendances mondiales, premier semestre 2015 et 2016, Genève; UNHCR: Une hausse sans précédent des déplacements forcés pourrait marquer l'année 2015 in http://www.unhcr.fr visité le 19 décembre 2015

${ }^{7}$ KNOMAD (Partenariat mondial pour les connaissances sur le développement et les migrations), Recueil des statistiques sur les migrations et les envois de fonds ; in http://wwwworldbank.org visité le 14 octobre 2016.
} 
conditions de vie en général, il s'ajoute de nouveaux facteurs tels que les catastrophes naturelles, la poursuite des études à l'étranger et des mouvements Nord/Sud de retraités dits migrations de «onforts».

Le deuxième est lié aux mouvements migratoires qui se sont plutôt régionalisés : aux mouvements Sud/Nord existent parallèlement d'importants flux Sud/Sud, à l'intérieur de l'Europe (des anciens pays socialistes vers l'Europe de l'Ouest). L'Afrique reste à cet égard le continent qui «produit» et héberge le plus grand nombre de réfugiés et des déplacés forcés en général. Il s'agit d'un constat confirmé par les récentes statistiques y relatives; les migrations Sud-Sud s'avèrent plus nombreuses que les migrations Sud-Nord. En 2013, plus de 38\% des migrants internationaux ont en effet migré d'un pays en développement vers un autre, alors que $34 \%$ ont quitté par contre un pays en développement pour s'installer dans un pays développé. Il en est de même pour les flux de réfugiés dans le monde, $86 \%$ d'entre eux sont accueillis dans des pays en voie développement ${ }^{8}$.

En conséquence, pour le troisième développement, les distinctions traditionnelles s'estompent: chaque territoire national peut changer de «statut» en fonction des objectifs ou du lieu de destination préférée par les migrants en passant ainsi de territoire d'émigration (pays de départ) à celui d'immigration (accueil) ou de transit.

Enfin, le quatrième développement consiste dans la modification des statuts des individus ou groupes concernés. Un réfugié politique peut devenir un migrant économique, un demandeur d'asile débouté peut devenir apatride et à cause du long séjour dans le pays d'accueil bénéficier d'un permis de travail pouvant lui ouvrir à terme la naturalisation, etc. ${ }^{9}$.

Figure $\mathrm{N}^{\circ} 1$ : Comparaison entre les migrants internationaux et les réfugiés, 2018

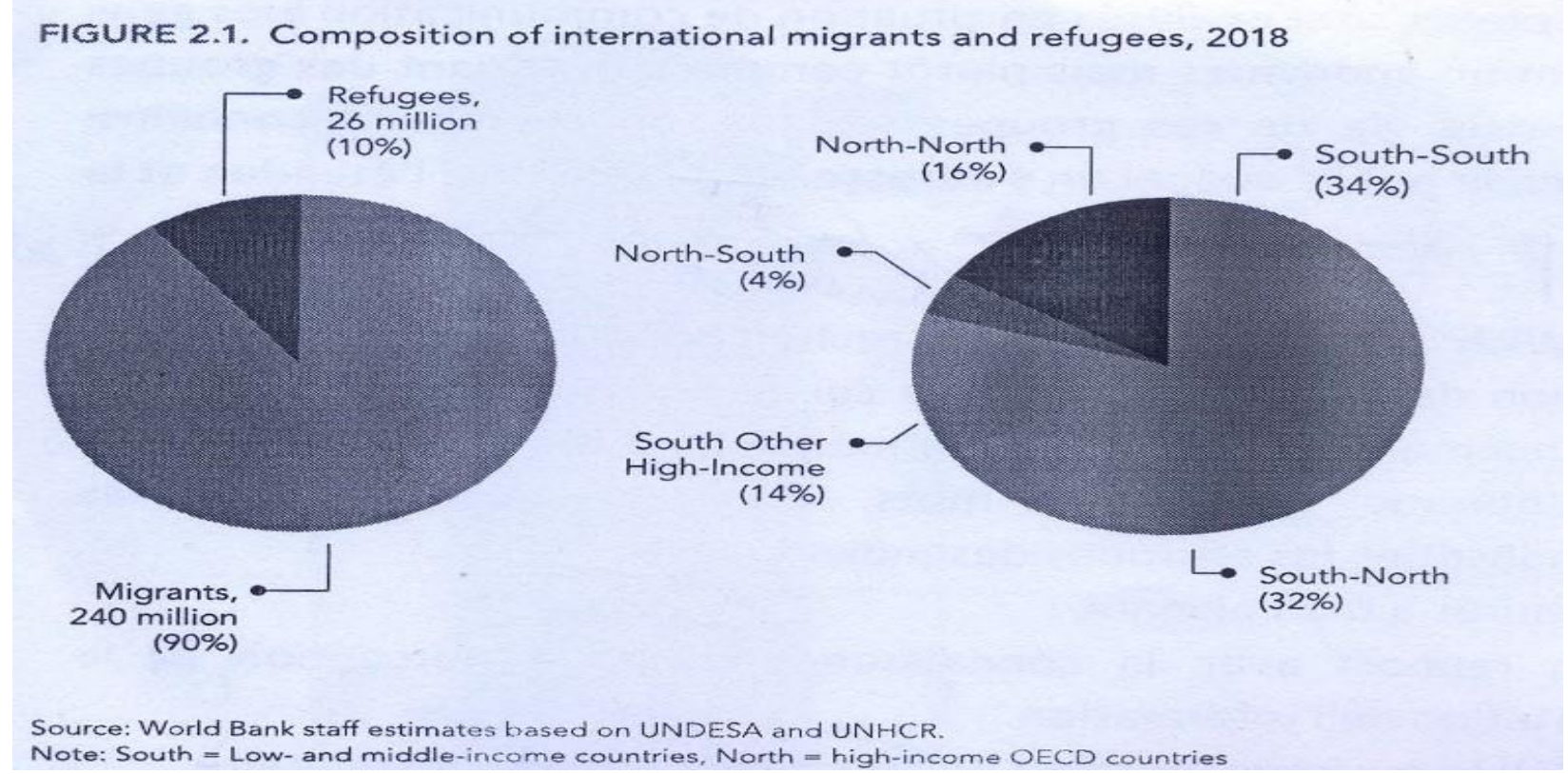

\footnotetext{
${ }^{8}$ KNOMAD, ibidem.
}

${ }^{9}$ Kirsch Olivier, op cit. pp. 12-14 


\section{Causes des migrations internationales}

\section{a) Un seul monde, de multiples opportunités}

Les causes du phénomène migratoire s'avèrent complexes à analyser dans la mesure où ils sont le fruit de plusieurs facteurs politique, économique, socioculturel, historique, etc. ${ }^{10}$ On comprend par facteurs économiques, la déprivation de moyens substantiels de vie tels que la pauvreté, le chômage endémique, qui décident bien des populations des pays en développement pour la plupart de quitter leur pays d'origine pour chercher meilleure fortune, une meilleure qualité de vie ailleurs et plus particulièrement dans les pays développés. Nous faisons face actuellement à un monde contrasté où, d'un côté, une grande population vit dans la pauvreté et la misère dans le Tiers-Monde tandis que de l'autre, les pays riches concentrent à la fois une grande partie de la richesse du monde et la gestion des ressources.

A cet égard, les récentes statistiques sur les migrations internationales et les renvois de fonds témoignent à la fois de l'importance accordée aux pays d'immigration et l'aide envoyée en retour par les immigrés dans leur pays d'origine: 23,2 millions de migrants sont d'origine subsaharienne, dont $26 \%$ environ vivent dans des pays de l'Organisation pour la Coopération au Développement Economique (OCDE) et 65,6\% dans des pays de la région ; les principaux pays d'origine des migrants étant la Somalie, le Burkina Faso, le Soudan, la République Démocratique du Congo, le Nigéria et la Côte d'Ivoire. Les envois de fonds des migrants originaires d'Afrique au sud du Sahara ont atteint 35 milliards de dollars en 2015, tandis que 4 milliards de dollars US en sont sortis en $2014^{11}$. Les envois de fonds («Remittances ») des pays à revenus élevés, «High-income Countries » (HIC) vers des pays à bas revenus, «Low-income countries » (LIC) atteindront 551 milliards de dollars US en 2019 (+4,7\% par rapport à 2018) et 597 milliards en 2021, dépassant ainsi l'investissement direct étranger, «Foreign Direct Investment» (FDI) en direction des mêmes pays à faible revenu, et donc du Tiers-Monde ${ }^{12}$. La Chine vient au premier rang avec 70,3 milliards de dollars US, les Philippines 35,1 milliards, l'Egypte 26,4 milliards, le Nigeria 25,4 milliards ; l'ensemble des pays de l'Afrique Subsaharienne a obtenu de ces envois de fonds de la part des migrants 49 milliards soit une augmentation de 5,1\%. En contrepartie, les pays d'origine des fonds sont naturellement des pays d'immigration, les Etats-Unis d'Amérique (27\%), l'Europe (12\%), le Canada (3\%), les pays membres du Conseil de coopération du Golfe (23\%), et les autres $(28 \%)$.

Sur le long terme, le Rapport Groundswell de la Banque Mondiale décline à cet effet trois facteurs qui feront augmenter l'envoi de ces fonds de pays à revenu élevé aux pays à revenu faible et d'émigration : le premier facteur est la différence de revenus entre ces deux groupes de pays, car la jeunesse de beaucoup de pays est frappée par la pauvreté, la misère et l'absence de perspectives. Le deuxième facteur est le changement démographique et le changement climatique car vers 2030, la population en âge de travailler dans les pays à bas revenu sera de 550 millions et ne pourra pas être absorbée par le marché du travail interne de leur pays de naissance tandis que

\footnotetext{
${ }^{10}$ Collins Alain (2013): Contemporary Security studies, Oxford: Oxford University Press, pp.138-139.

${ }^{11}$ KNOMAD, op. cit.

12 Banque Mondiale (2019) : Rapport de la Banque Mondiale. Leveraging Economic migration for development. A briefing for the World Bank Board, Washington: World Bank Group ou http://www.knomad.org, visité le 10.01.2020.
} 
vers 2050, 143 millions de personnes seront des réfugiés climatiques (40 millions en Afrique Subsaharienne, 40 millions en Asie du Sud et 17 millions en Amérique latine et enfin le troisième facteur étant le flux de migration et de réfugiés subséquent ${ }^{13}$.

\section{b) Politique, Guerres, Changement climatique}

D'après le Centre de Recherche sur les causes des guerres et conflits dans le monde de Hambourg (Allemagne), «Hamburger Arbeitsgemeinschaft für KriegsUrsachenForschung (AKUF)», il existe actuellement 27 guerres et conflits armés dans le monde dont 8 au Moyen et Extrême Orient, 10 en Afrique, 7 en Asie, 1 en Europe et 1 en Amérique centrale et latine respectivement ${ }^{14}$. D'autres sources font état de 49 guerres et conflits à travers le monde en fonction du niveau d'intensité et du nombre des victimes ${ }^{15}$. Les parties en conflit visent également à atteindre un objectif devenu aujourd'hui stratégique, à savoir, d'un côté, porter les batailles déstabilisatrices dans les zones urbaines ou la capitale du pays où se concentrent à la fois le pouvoir politique et économique, et de l'autre, le contrôle des populations en période de guerres civiles. Il s'agit pour une opposition armée d'ôter l'Etat d'une partie de sa souveraineté sur la population tandis que pour les troupes régulières, il faut empêcher une guérilla de contrôler une frange de la population pouvant servir de boucliers humains, de sources de ravitaillement en moyens humains et matériels pour la poursuite de la guerre. Ce contrôle direct de la population se traduit en termes de destructions humaines, de déplacements forcés de populations pouvant devenir une source de revenus vu l'aide humanitaire qui parfois s'ensuit et ainsi pour les radicaux ou «hardliners »structurer toute la société uniquement autour des enjeux du conflit interne ${ }^{16}$.

Dans d'autres cas, les flux de réfugiés sont produits par un système politique ou une situation de conflits violents stigmatisant une partie de sa population. C'est le sort de beaucoup de pays en conflits violents. Dans cet ordre d'idées, les réfugiés africains sont encore estimés à 208.000 en $1965^{17}$, ils atteignent 18 millions en 2019 (soit 26\% des réfugiés dans le monde), bref une progression vertigineuse qui témoigne de la gravité du phénomène et de ses causes multidimensionnelles. L'ensemble des pays en voie de développement héberge $86 \%$ des réfugiés sous mandat du HCR parmi lesquels les pays sous-développés («Least Developed Countries») ont accordé l'asile à 4,2 millions de réfugiés dans le monde. A titre d'exemple, l'Afrique accueille $1 / 3$ des réfugiés dans le monde soit 6,3 millions tandis que l'Europe n'a ouvert ses portes qu'à $13 \%$ d'entre eux soit 2,6 millions $^{18}$.

La Convention de l'Organisation de l'Unité Africaine (OUA devenue Union Africaine depuis 2002) du 10 septembre 1969 et entrée en vigueur le 20 juin 1974 constitue une réaction aux aspects propres des problèmes posés par les réfugiés en Afrique. Ainsi, le statut de réfugié

${ }^{13}$ Voir également Banque Mondiale (2018) : Groundswell : Preparing or internal climate Migration, Washington DC : World Bank Group.

${ }^{14} \mathrm{http}: / / \mathrm{www}$.akuf.de visité le 11.01.2020.

${ }^{15}$ www.fr.wikipedia.com visitée le 15.12.2019.

${ }^{16}$ De Montclos M. A.P «Les flux de réfugiés : un enjeu politique au Sud comme au Nord», La Chronique du CEPED, Octobre-Décembre 1998, №31, p. 1.

${ }^{17}$ Presque à la création de l'Organisation de l'Unité Africaine (OUA) en 1964, voir Scandinavian Institute of Africa Studies, Refugee problem in Africa, Uppsala, 1967, pp.14-15.

${ }^{18}$ Global Trends, Forced Displacement en 2015, http://www.unhcr.org consulté le 15 novembre 2016. 
s'applique également à «toute personne qui, du fait d'une agression, d'une occupation extérieure, d'une domination étrangère ou d'événements troublant gravement l'ordre public dans une partie ou dans la totalité de son pays d'origine ou du pays dont elle a la nationalité, est obligée de quitter sa résidence habituelle pour chercher refuge dans un autre endroit à l'extérieur de son pays d'origine ou du pays dont elle a la nationalité.» ${ }^{19}$

L'élargissement du champ des critères conduit in fine à reconnaître le statut de réfugié sur une base dite «prima facies» sans que les candidats puissent démontrer leur persécution. Ce traitement pragmatique a l'avantage de régler sur le plan humanitaire et juridique des mouvements massifs et désordonnés de réfugiés consécutifs à des conflits violents sans qu'il y ait possibilité matérielle de mener des interviews individuelles.

Actuellement, sur les 70,8 millions de personnes déplacées forcées, le premier groupe est composé de réfugiés soit 25,9 millions (soit 500.000 personnes de plus que l'année précédente 2017). Ce chiffre comprend en réalité 19,9 millions réfugiés dans le monde sous la protection du HCR et 5,5 millions de réfugiés palestiniens relevant de la responsabilité de l'Office de secours et des travaux des Nations Unies pour les réfugiés de Palestine dans le Proche-Orient (UNRWA).

Le second groupe est constitué de demandeurs d'asile, autrement dit des personnes se trouvant en dehors de leur pays d'origine et qui bénéficient certes d'une protection internationale, mais demeurent dans l'attente d'une réponse à leur requête d'obtention du droit d'asile en vue d'accéder au statut de réfugié. En 2018, elles étaient estimées à 3,5 millions à travers le monde. Ce droit d'asile est proclamé par la Déclaration Universelle des droits de l'Homme de 1948 dont l'article 14 stipule que «devant la persécution, toute personne a le droit de chercher asile et de bénéficier de l'asile en d'autres pays ». Depuis 2013, les Etats européens ont conclu un régime d'asile commun qui intègre la protection subsidiaire au profit des personnes menacées dans leur pays d'origine mais dont la situation ne répond pas à la définition du statut de réfugié. Elles peuvent ainsi bénéficier de l'asile au titre d'un régime dit de «protection subsidiaire » lorsqu'il existe de motifs sérieux et avérés qu'elles peuvent courir dans leur pays un risque réel de subir la peine de mort, la torture ou des traitements dégradants, ou une menace grave et individuelle contre leur vie en raison d'une violence aveugle résultant d'une situation de conflit armé interne ou international ${ }^{20}$.

Le troisième groupe et de loin le plus important est estimé à 41,3 millions de personnes forcement déplacées comprenant également des individus déplacés vers d'autres régions au sein de leur propre pays, et qui forment une catégorie appelée «déplacés internes » ou «Internal, Displaced People » (IDP). Parmi ces personnes obligées d'abandonner leurs foyers, la majorité d'entre elles viennent des zones de conflits en Afrique centrale, au Moyen-Orient, en Colombie et dans les pays de l'ex-Yougoslavie. Plus de la moitié des réfugiés à travers le monde soit $68 \%$

\footnotetext{
${ }^{19}$ Article 1, alinéa 2 de la Convention de l'OUA régissant les aspects propres aux problèmes des réfugiés en Afrique de 1969.

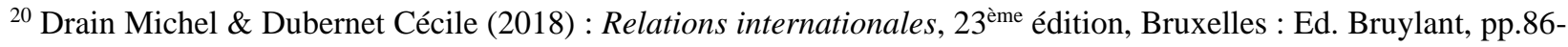
87.
} 
viennent de quatre pays, à savoir la Syrie (6,3 millions), l'Afghanistan (2,6 millions) et le Sud Soudan (2,4 millions) qui devance la Somalie $(986.400)^{21}$.

\section{c) Catastrophes naturelles}

Les changements climatiques poussent les pays d'accueil à considérer une autre catégorie de déplacements de population, les réfugiés de l'environnement dits «réfugiés climatiques ou écoréfugiés». Le terme de «réfugiés environnementaux» fut utilisé pour la première fois en 1985 par l'Egyptien Essam El Hinnaoui qui les désigne comme «ceux qui ont été forcés de quitter leurs habitations traditionnelles, d'une façon temporaire ou permanente, à cause d'une perturbation environnementale nette (naturelle ou provoquée par l'homme) qui a mis en danger leur existence et/ou sérieusement affecté leur qualité de vie» ${ }^{22}$.

Ces catastrophes naturelles sont de nature diverse: elles peuvent être soudaines et brutales comme des séismes, cyclones, inondations pouvant produire des dommages à caractère irréversible tel que la montée du niveau de la $\operatorname{mer}^{23}$, érosion des fleuves, désertification, déforestation et se soldent souvent par des morts et des déplacements de populations. Des accidents industriels peuvent être considérés comme des catastrophes naturelles (glissement de terrains, coup de grisou, explosions d'usines etc.). Actuellement, des milliers de personnes sont déjà déplacées par des marées de tempêtes dans des pays comme le Bangladesh, une situation qu'on observera encore dans les années à venir dans d'autres pays puisqu'elle est due au changement climatique ${ }^{24}$.

Au-delà de la compassion et de la générosité que soulèvent ces hasards naturels, il n'en reste pas moins que sur le plan juridique, il s'avère difficile de les reconnaître comme fait juridique pouvant ouvrir le droit au statut de réfugié. L'état actuel du droit international ne permet pas de catégoriser un groupe de réfugiés climatiques même si dans la pratique, les Etats prennent en compte cette catégorie et par conséquent dépassent ainsi un blocage caractéristique du système international actuel à savoir la non-ingérence dans les relations interétatiques. Le sens de réfugié est conçu de façon restrictive malgré les protocoles additionnels intervenus depuis 1951 en vue de s'adapter aux différentes réalités et exclut de son registre déplacés internes ou réfugiés internes, refugiés climatiques au-delà de la nécessité bien fondée de prendre en charge des victimes $^{25}$. Ce qui compte le plus reste en réalité le fait qu'ils mettent en danger le fonctionnement normal d'une communauté et par conséquent, bien que le HCR ait été à l'origine créé pour diriger et coordonner l'action internationale visant à protéger les réfugiés a dans les faits étendu cette mission aux apatrides depuis la fin des années 1990 et puis aux personnes déplacées à l'intérieur de leur Etat d'origine.

\footnotetext{
${ }^{21}$ Rapport HCR 2018 ; voir également le site de la Deutsche Welle ; www.dw.com.

${ }^{22}$ Voir Rapport pour le PNUE en 1985.

${ }^{23}$ Le Tsunami de 2003.

${ }^{24}$ Swain Aschok,"Environmental migration and conflict dynamics: Focus on developing regions", Third World Quarterly, Vol. 17, N5, 1996, pp.959-973; voir également HCR, op. cit., p.2.

${ }^{25}$ Lemaitre Th., «Réfugiés environnementaux, de quoi parle-t-on? », http://www.vuessurlemonde.com visité le 15 novembre 2015 .
} 


\section{d) Des politiques migratoires restrictives}

S'il est difficile voire indécent de présenter les politiques migratoires restrictives des pays occidentaux particulièrement comme causes des migrations illégales et forcées, force est de constater que la restriction dans les procédures d'octroi du visa, particulièrement après la chute du mur de Berlin marquant ainsi la fin de la guerre froide, n'a pas pour autant arrêté les flux migratoires à travers le monde. Certes, il existe actuellement une séparation rigide entre d'un côté les services de gouvernements, d'organismes internationaux et autres qui invitent des officiels étrangers au voyage, et de l'autre, les services d'immigration, au point que cette division des tâches ne garantit pas pour autant à ces derniers l'octroi du visa. Cette rigueur décourage sans doute des velléités de départ, mais les candidats à l'émigration useront de toutes les combines pour quitter leur pays et régions d'origine, avec pour résultat inattendu que 900.000 personnes traverseraient illégalement les frontières internationales ${ }^{26}$.

\section{Typologie des migrants internationaux}

\section{a) Demandeurs d'asile et Réfugiés}

Aux termes de la Convention du 28 juillet 1951 relative au Statut des réfugiés, on entend par réfugié, «Toute personne qui, craignant avec raison d'être persécutée du fait de sa race, de sa religion, de sa nationalité, de son appartenance à un certain groupe social ou de ses opinions politiques, se trouve hors de son pays d'origine et qui ne peut ou, du fait de cette crainte, ne veut réclamer la protection de ce pays» ${ }^{27}$. Cette définition était limitée aux réfugiés d'avant 1951 , faisant donc allusion aux mouvements de déplacement des populations suite à la deuxième guerre mondiale. Il aura fallu donc le Protocole additionnel de New York relatif au Statut des réfugiés du 31 janvier 1967 pour élargir ladite définition à toute personne répondant à la qualité de réfugié.

Ce protocole innove à cet égard en éliminant toute référence à l'année 1951 et à des restrictions d'ordre géographique. Concrètement, la Convention de Genève de 1951 se limitait aux transfuges quittant l'Europe de 1'Est pour l'Ouest, généralement des hommes politiques et intellectuels demandant asile dans les pays occidentaux. En outre, cette convention limitait son application au territoire européen. Il devient donc applicable à tous les pays y compris ceux qui ne font pas partie à la convention, une innovation certes introduite dix ans après la Convention de 1951 qui ne devrait en aucun cas prétendre régler la problématique des réfugiés sur tous les continents bien que la guerre froide structurait les relations internationales à l'époque.

\section{b) Migrants de travail dits migrants économiques}

Quant aux réfugiés économiques, encore faut-il isoler cette cause et en déterminer des variables dépendantes, car les pays et régions potentiellement en proie à des conflits internes sont ceux où le progrès politique et économique s'avère encore bas. Ces aspects étant intimement liés, il

\footnotetext{
${ }^{26}$ Collins Alan : Op. Cit., p. 138.

${ }^{27}$ Article 1.
} 
s'avère éminemment urgent de distinguer les questions structurelles de questions conjoncturelles. Des déséquilibres entre pays en matière de développement font que des individus émigrent à la recherche de meilleures conditions de vie.

Ceci dit, au niveau juridique et conceptuel, les réfugiés n'ont d'autre choix que de quitter leur pays, contrairement aux migrants. Les migrants économiques jouissent toujours de la protection de leur pays d'origine et peuvent, s'ils le veulent, y retourner en toute quiétude alors que le réfugié a quitté son pays d'origine pour chercher protection et sécurité sous un autre Etat, et ne peut plus y retourner.

\section{d) Migrants environnementaux}

Les migrants ou déplacés environnementaux sont encore peu nombreux, 38 millions en 2011 et pourraient atteindre les 150 millions en 2050. Cet aspect a marqué les rencontres préparatoires du récent Sommet mondial de Paris sur les changements climatiques (COP 21) du 30 novembre au 12 décembre 2015. La pluralité des termes utilisés traduit la diversité quant au statut juridique de ce type de mouvements de populations: «déplacés», «réfugiés», «migrants environnementaux» sont autant de termes qui traduisent différents cas de figure résultant des aléas climatiques : dégradation progressive ou brutale de l'environnement, changement climatique dans le monde, inondations au Bangladesh, au Mozambique, désertification au Sud du Sahara, explosion de gaz méthane au Cameroun, menaces posées par le dépôt des déchets nucléaires, catastrophes industrielles, etc. ${ }^{28} \mathrm{Si}$ les caprices environnementaux sont à l'origine de déplacements de populations, leur prise en compte dans les phénomènes migratoires est récente comme nous l'avons vu.

\section{e) Regroupement familial}

Appelé également migration sociale pour certains, le regroupement familial désigne les déplacements de personnes qui entendent rejoindre des membres de leur famille installés temporairement ou définitivement dans un pays étranger (conjoint, enfants, ascendants, etc.). Cette forme de migration se produit souvent en cas de demande acceptée d'asile, de séjour permanent fondant ainsi le regroupement familial, lequel peut avoir des conséquences sociales dans la mesure où par exemple le taux d'emploi des femmes ayant bénéficié du regroupement familial est plus élevé dans leur pays d'accueil que dans leur pays d'origine, la migration pouvant constituer pour elles un facteur d'émancipation ${ }^{29}$.

\section{f) Migrants de confort}

La migration de confort concerne une partie de migrants (retraités, rentiers, personnes âgées, etc.) qui optent pour venir s'installer dans un pays en vue de profiter de sa qualité de vie en fonction de leur souhait ou état de santé (calme, climat, raisons sanitaires, etc.). Ces migrants de confort

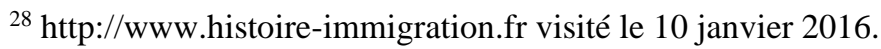

${ }^{29}$ Kirssch Olivier, op, cit, p. 20. 
gardent le lien avec leur pays d'origine dont ils restent ressortissants (expatriés britanniques, Allemands sous le soleil de France, d'Espagne, des Américains au Costa Rica, etc.) tout en bénéficiant d'un cadre de vie de leur choix, et, en fonction des politiques migratoires, d'un régime fiscal favorable tel que par exemple l'exonération d'impôts à condition de justifier des revenus mensuels supérieurs à $5.000 \$$ ou bien d'une rente à vie $\mathrm{e}^{30}$.

\section{g) Apatrides}

Quant à l'apatride, il s'agit d'un étranger qui ne jouit pas de droits réservés aux nationaux soit parce qu'il n'en a pas la nationalité, soit parce qu'il en est dépourvu. L'apatride se définit comme une personne qu'aucun Etat ne considère comme son ressortissant par application de sa législation $^{31}$. Il s'agit d'un problème d'une portée internationale dans la mesure où selon le Rapport Global Trends 2015 du HCR, plus de dix millions de personnes se trouvaient dans une situation d'apatridie dont un tiers serait des enfants, bien que les chiffres communiqués par les Etats fassent état par contre de 3.300.000 d'individus apatrides dans 72 pays dans le monde ${ }^{32}$. Cette disparité des données chiffrées explique en partie la pluralité des facteurs à l'origine de l'apatridie.

En effet, les causes de l'apatridie sont multiples et résultent de plusieurs facteurs tels que la succession d'Etats, des législations restrictives en matière de transmission de la nationalité telles que celles qui frappent particulièrement les femmes qui ne peuvent pas ainsi transmettre leur nationalité à leurs enfants surtout ceux nés en dehors de leur pays d'origine, des obstacles simples comme la non déclaration des naissances, la discrimination à l'égard des femmes et la loi sur les mariages, la discrimination raciale et ethnique, la renonciation à la nationalité sans acquisition préalable d'une autre nationalité.

\section{Enjeux et défis des migrations internationales}

\section{a) L'accueil et le rétablissement}

Les déplacements de migrants affectent tant ceux qui sont obligés de quitter leur patrie ou pays d'origine en général que les populations d'accueil pour lesquelles tolérance, compassion et solidarité s'avèrent plus que nécessaires ${ }^{33}$. Dans les pays d'accueil, la venue des réfugiés pose diverses questions dans la mesure où il s'agit d'une vague d'individus, étrangers arrivant massivement dans un pays lointain avec un environnement politique, économique, culturel complètement différent. Surtout en période de crise économique ou de difficulté de partage de la richesse nationale, un changement d'attitude s'instaure dans l'opinion, se traduisant dans un

\footnotetext{
${ }^{30}$ De Legge, «Le Costa Rica, le refuge des retraités américains », in http://www.journaldunet.com visité le 12 novembre 2016.

${ }^{31}$ Convention relative aux statuts des apatrides du 28 septembre 1954, article 1, alinéa 1.

${ }^{32}$ UNHCR, UNHCR Global trends Displacement the new 21 st century challenge, Genève, 2012, p.1.

${ }^{33}$ Propos d'Antonio Guterres, HCR, op.cit., pp. 2-3.
} 
premier temps par la notion de «seuil de tolérance», «on ne peut pas accueillir la misère du monde», etc., et la politisation accrue du phénomène migratoire.

La pression augmente sur les pays qui accueillent les réfugiés; l'attitude des gouvernements et des sociétés d'accueil des réfugiés, en majorité syriens, aura été dans l'immédiat d'une générosité sans précédent faisant fi des législations restrictives en la matière. Ce flux suscite par contre un ressentiment de la part des populations d'accueil et une volonté de politiser le phénomène migratoire ${ }^{34}$.

Actuellement, plus de 16 millions de personnes dont s'occupe le HCR n'ont pas pour la plupart le statut de réfugiés. Ces derniers restent dans les pays limitrophes des pays sous-développés où ils ne peuvent pas bénéficier de droits dont les nationaux ne jouissent pas. Une visite récente dans le camp de réfugiés de Meheba au Nord de la Zambie en octobre 2015 démontre à suffisance cette situation de précarité. Les réfugiés vivent dans différents quartiers, parfois des groupes nationaux encore ennemis au début, par exemple des Tutsi et des Hutu du Rwanda et du Burundi vivant côte à côte. Il aura fallu des menaces directes proférées par les autorités zambiennes de les renvoyer dans leur pays d'origine pour qu'ils cessent de répliquer les attitudes violentes du Rwanda pendant le génocide en 1994 et la guerre civile au Burundi en $1993^{35}$.

Un des baromètres de l'état des réfugiés dans le monde consiste à mesurer le nombre de retours volontaires à savoir une mesure du nombre de réfugiés pouvant rentrer chez eux sans courir aucun risque; ce dernier est estimé à 84.000 personnes par rapport à 107.000 sur la même période il y a un an (janvier-juin 2014). Les chances pour un réfugié d'aujourd'hui de rentrer dans son pays n'ont jamais été aussi faibles depuis 30 ans $^{36}$.

\section{b) Impact de la présence des réfugiés dans les rapports interétatiques}

En recherchant protection et sécurité dans un autre Etat, la présence des réfugiés exerce un impact évident à divers niveaux des Etats et des sociétés. Premièrement dans les rapports interétatiques, un aspect est de reconnaître le drame humain et accorder l'asile à des groupes en détresse, un autre qui peut en découler indirectement est de contribue à l'internationalisation du conflit interne de leur pays d'origine. Aussi peut-il naître des tensions entre pays d'accueil («hosting state») et pays d'origine («sending state») comme le démontre actuellement les relations entre régimes au pouvoir dans les pays de la Région des Grands Lacs depuis leur accession à l'indépendance.

En principe, ces tensions ne devraient pas exister tant le statut de réfugié constitue une situation juridique clairement encadrée par différents instruments nationaux, régionaux et internationaux qui définissent les droits et devoirs des Etats hôtes et des réfugiés. Ainsi, le réfugié se distingue-til d'autres situations proches dans lesquelles un individu se trouve à l'étranger ou à l'intérieur de son pays. L'étranger ayant franchi une frontière internationale s'emploie à obtenir le statut de réfugié, il est alors qualifié de «demandeur d'asile». Il se distingue de l'émigrant ordinaire qui,

\footnotetext{
${ }^{34}$ Echec jusqu'aujourd'hui de la politique des quotas en Europe, la question de l'immigration s'invite dans la campagne américaine, tri de réfugiés sur la frontière avec la Hongrie alors qu'en réalité la Turquie (1,8 millions) et le Liban (209 réfugiés pour 1000 habitants) accueillent le gros des réfugiés syriens pour la plupart.

${ }_{35}^{35}$ Propos des responsables des camps, jeudi 04 novembre 2015.

${ }^{36} \mathrm{HCR}$, op. cit, p. 2
} 
tout en séjournant à l'étranger ne rompt pas avec son pays d'origine. Au-delà de la nature juridique du sens de réfugié, il existe d'autres situations où des individus pris isolément ou des groupes se retrouvent en dehors de leur pays d'origine dont il est question dans la suite du travail. Deuxièmement, au niveau interne, l'accueil des réfugiés a des conséquences également dans le pays d'accueil. L'octroi de l'asile à un étranger accorde à ce dernier le statut de réfugié et par conséquent le bénéfice des droits et le respect des devoirs y afférents: des droits patrimoniaux (exercice d'une profession, droit à la propriété, etc.), des droits extrapatrimoniaux (regroupement familial par exemple). Par la suite, l'immigrant devra pouvoir s'intégrer dans cette nouvelle vie du pays d'accueil.

Troisièmement, la question des migrations internationales fait l'objet d'un débat sur le plan sécuritaire. Pour les études de sécurité, le phénomène migratoire s'analyse en termes de menace à la sécurité du pays hôte sur divers plans: en fait de sécurité politique, une migration forcée, incontrôlée, « undocumented » dit-on, porte atteinte à la sécurité des frontières nationales et donc à la souveraineté d'un Etat. Sur le plan de la sécurité économique et sociale, ce phénomène incontrôlé exerce in fine une menace à la fabrique sociale et économique des Etats en affectant leur ordre social et le taux de criminalité, même quand ils acceptent des emplois sous qualifiés, mal rémunérés. De plus, les migrants sont vus comme une menace au mode de vie, à la culture, à la religion du pays d'accueil ${ }^{37}$.

Les partisans de la gestion de la diversité par contre s'emploient à comprendre le phénomène migratoire, le mode de vie des populations immigrées de différentes générations quant à son apport à l'économie, à la société du pays d'accueil et in fine faire de la population immigrée, quels que soient leur culture, leur mode de vie une chance pour le pays hôte : des populations immigrées dont certains membres ont déjà acquis avec le temps la nationalité, des travailleurs et des consommateurs à la fois contribuant à la prospérité économique du pays, qui s'adonnent à des tâches que les nationaux de souche ne veulent pas exécuter soit à cause de leur dureté, soit des horaires de travail, etc. Gérer la diversité consistera donc à la reconnaitre et non la bannir, accepter cette réalité dans la vie quotidienne, au travail, en famille, à l'école, etc. Il en résulte des études et recherches sur la diversité et son management, des théories sont développées, des concepts définis, des dimensions analysées (âge, handicap, ethnicité, genre, religion, différentes orientations, etc.), et de nouvelles formes de compétence sociale découvertes et enrichies ${ }^{38}$.

Enfin et quatrièmement, une meilleure intégration économique et sociale de la diaspora peut conduire à une désescalade des conflits dans les pays d'origine. Une étude faite en 2013 sur les 500 membres de la diaspora rwandaise en Suède montre qu'une meilleure intégration économique dans le pays d'accueil peut diminuer l'implication de ces derniers dans la polarisation ethnique, religieuse et autre, contribuant ainsi à la réduction des tensions politiques $^{39}$.

\footnotetext{
${ }^{37}$ Collins Allan : Op. Cit., pp. 138-139.

${ }^{38}$ Bendl Regine / Hanappi-Egger Edeltraud \& Hofmann Roswitha (2012) : Diversität und Diversitätsmanagement, Wien : Facultas Verlag AG ; Garner-Moyer Hélène (2006) : "Gestion de la diversité et enjeux de la gestion des ressources humaines »; in Management et Avenir, 2006/1 ( $\left.\mathrm{N}^{\circ} 7\right)$, pp. 23-42.

${ }^{39}$ Rolland Cécilia (2013) : Decreased Polarization through economic integration ? A study of ethnic polarization of members of the Rwandese diaspora 19 years after the genocide, Master thesis in Peace and conflict studies, Uppsala University : DPCR.
} 
Ces quatre cas de figure susmentionnés s'appliquent en grande partie aux pays développés ayant un ordre politique, économique, culturel et autre fonctionnel. Or la plupart des migrants restent dans les pays de leur région d'origine, dans les pays du Tiers-Monde. Ces derniers éprouvent encore des difficultés pour sortir du sous-développement et par conséquent de pouvoir intégrer physiquement, politiquement tout le pays, garantir des droits à des étrangers qu'ils accueillent sur leur sol. Ils ne peuvent pas offrir à des populations étrangères, forcement déplacées, ce qu'elles ne peuvent pas offrir aux leurs. Dans les pays d'Afrique et du Tiers-Monde en général, les Etats éprouvent encore des difficultés à gérer la diversité dans tous ses aspects (ethnie, religion, région, langue, groupe d'intérêt, etc.) et le grand défi reste de ramener tous ces différents facteurs de la diversité d'un Etat dans un système soit-il politique, économique, culturel, administratif, etc. ${ }^{40}$

Tableau $\mathrm{N}^{\circ} 1$ : Typologie des migrants et des déplacés forcés

TABLE 1.1. Typology of Migration and Forced Displacement

\begin{tabular}{|c|c|c|}
\hline & Internal & International \\
\hline $\begin{array}{l}\text { Migration } \\
\text { (economic or voluntary) }\end{array}$ & $740-763$ million & Migrants 240 million \\
\hline \multirow[t]{2}{*}{ Forced } & Climate-driven IDPs 20 million & $\begin{array}{l}\text { Migrants (Climate-driven or } \\
\text { mixed flows) }\end{array}$ \\
\hline & FCV driven IDPs 41 million & Refugeess 26 million* \\
\hline
\end{tabular}

\section{La Région des Grands Lacs Africains : de flux croisés de déplacements forcés}

\section{Une migration économique lointaine et ordonnée}

Pendant la période coloniale, des mouvements de populations ont caractérisé les pays de la région des Grands Lacs et particulièrement le Burundi: suite à des tentatives d'invasion étrangères, aux pratiques autoritaires des pouvoirs en place pendant la colonisation allemande (1886-1918) et la présence belge (1919-1962), à des catastrophes humanitaires dues à la

${ }^{40}$ National Intelligence Council (2008) : « Democratization in Africa : What Progress toward Institutionalization? » Conference Report, Februar ; Teshome Wondwosen (2009) : «Opposition Parties and the Politics of Opposition in Africa : A critical Analysis »; in International Journal of Human and Social Sciences, 4 :5, pp. 287-301. 
sécheresse et aux famines, une partie de la population, mâle pour la plupart, a été obligée d'émigrer vers les pays limitrophes. Un indice évident de cette trace ou présence humaine est la langue et la culture burundaise dans lesquelles se reconnaît une partie de la population des différents pays limitrophes.

Pendant la colonisation belge, des mouvements migratoires de la population burundaise s'observent en direction des pays limitrophes, premièrement vers le Congo belge (actuelle République Démocratique du Congo). Ces mouvements ont pour objectif d'un côté de fournir une main-d'œuvre laborieuse et peu coûteuse devant prester dans les riches mines du Katanga et de l'autre de trouver une solution au problème de deux ordres posé par le processus de recrutement des travailleurs dits indigènes dans les entreprises européennes : Avant la crise économique des années 1930, le processus de recrutement des travailleurs des entreprises privées et des administrations publiques se faisait par le biais d'agents recruteurs en vue d'obtenir la presque totalité de la main-d'œuvre locale dont elles avaient besoin. Il ne fallait donc pas que ce processus de recrutement se réalise par la contrainte exercée sur les nationaux à laquelle ces recruteurs recourraient souvent. Ensuite, les exigences en main d'œuvre des employeurs privés et des administrations publiques entraînaient des prélèvements excessifs en nombre d'hommes valides dans leur communauté. Il était donc question que l'embauche des travailleurs congolais ne conduise pas à un déséquilibre de la structure démographique dans les communautés locales. Ainsi, une Commission spéciale pour l'étude du problème de la main-d'œuvre instituée par le gouvernement belge en 1924 propose un pourcentage maximal du nombre d'hommes adultes valides devant être recrutés dans les entreprises européennes sans mettre en danger l'équilibre démographique des collectivités indigènes, entendons ici le rapport hommes/femmes. Ce maximum sera fixé par une mission envoyée par le Ministère des Colonies à neuf dixièmes d'hommes adultes devant rester normalement dans les villages ${ }^{41}$.

Deuxièmement, en direction des ex-colonies britanniques, particulièrement la Tanzanie et l'Ouganda qui avaient besoin d'une main d'œuvre abondante pour leurs plantations théicoles. La population d'origine burundaise en Ouganda serait estimée à un million et demi. Certes, elle garde des relations assez lointaines avec le Burundi mais avec néanmoins un désir de renouer des liens économiques et culturels assez fonctionnels.

Dans les deux cas susmentionnés, d'autres facteurs ont joué un rôle non moins important, à savoir certaines pratiques de la période coloniale telles que des corvées multiformes, la perception de l'impôt de capitation, la chicote (le fouet) pratiquée par les colons belges ou alors l'attrait de salaires plus intéressants pratiqués dans les plantations des colons britanniques et des indiens en Tanzanie et en Ouganda. De 1949 à 1959, 24.799 Burundais avaient émigré vers le Congo belge, tandis que 183.482 vers les territoires britanniques ${ }^{42}$. Avant cette période, le Rapport de l'Administration du Ruanda-Urundi de 1929 fait état de 320 Barundi ayant émigré temporairement vers le District du Kivu (Est de la RD Congo); tandis qu'au 31 décembre de la

\footnotetext{
${ }^{41}$ Barret Francois (1952) : Histoire du travail, Col. Que sais-je ? №164, Paris : PUF, pp.51-58 ; Convention №50 du 20 juin 1936 relative au recrutement des travailleurs indigènes approuvée par la loi du 10 septembre 1947 , BO 1948, p. 1023 ; Ministère des Colonies (1932) : Rapport sur l'Administration belge du Ruanda-Urundi, p. 98.

${ }^{42}$ Rapport sur l'Administration belge du Ruanda-Urundi, 1959.
} 
même année, les effectifs des Banyarwanda et des Barundi présents au Katanga étaient de 3.573 hommes, de 1.396 femmes et de 726 enfants.

Des entretiens personnels effectués à Lubumbashi en mars 2014-2016, il ressort qu'il existe un groupe de Congolais originaires du Rwanda et du Burundi et qui, pour la plupart, gardent un souvenir lointain, une connaissance ambiguë de leurs origines en dépit d'un attachement affectif remarquable. Ce sont des personnes de la première jusqu'à la troisième génération, qui ne sont plus retournées dans leur pays d'origine, et les crises récurrentes aidant, elles ont été découragées de cultiver davantage leurs relations antérieures avec des parentés restées au pays.

Cette émigration en direction de l'Ouest au sud de l'actuelle RD Congo d'un côté, et vers les excolonies britanniques à l'Est d'autre part, s'explique sans doute par des facteurs géographiques de la région (position à cheval sur le Congo et l'Afrique orientale) et historiques (colonisation belge d'un côté et britannique de l'autre), démographiques (pays surpeuplés au Rwanda et au Burundi). Cette logique explique également des phénomènes migratoires internes et régionaux dans les contrées limitrophes du Burundi.

La mise en valeur de la plaine de l'Imbo (au Nord-Ouest du Burundi) donne lieu à un flux migratoire prélude au développement de cette région. En effet, d'après l'historien de la Région des Grands Lacs africains, Jean-Pierre Chrétien, «l'histoire agricole de l'Imbo et l'histoire de son peuplement ne commencèrent que vers les années 1940 avec les débuts de la mise en valeur humaine importante. La plaine de la Ruzizi voit la mise en place dans les années 50 de paysannats dits indigènes» ${ }^{43}$. A la suite de la création des paysannats dits indigènes, des paysans originaires des régions peuplées de Muramvya, Kayanza et Ngozi se déplaçaient vers la plaine de l'Imbo en vue d'occuper des terres vacantes, tandis que d'autres y venaient occuper temporairement un emploi rémunéré dans la compagnie d'exploitation cotonnière Ruzizi ou dans des plantations de coton. Si parmi ces derniers certains regagnaient leur région d'origine, d'autres s'y installaient également et définitivement avec leur famille.

\section{Des crises politiques récurrentes et des flux de migrations forcées}

Situé dans la Région des Grands Lacs Africains, une région particulièrement instable depuis les cinquante dernières années, le Burundi connaît une grande diversité de migrations forcées, en réunissant sous son territoire toutes les catégories de personnes sous mandat du HCR : apatrides, rapatriés, déplacés internes, demandeurs d'asile, réfugiés, etc. Avec les crises récurrentes des cinquante dernières années, le Burundi accueille sur son sol en même temps «qu'il produit » également des demandeurs d'asile venant ou allant dans les pays limitrophes à savoir la RD Congo, le Rwanda, la Tanzanie et l'Ouganda principalement.

Le premier mouvement de déplacés forcés concerne les réfugiés tutsi rwandais fuyant le Rwanda suite à la «révolution sociale de 1959». Ces derniers s'installent dans la capitale Bujumbura, et dans des camps à l'Est du pays: en 1964, ils étaient estimés à 35.000 à Mugera, 10.000 à Murore,

${ }^{43}$ Chrétien J-Pierre : «La crise écologique de l'Afrique orientale au début du XXème siècle. Cas de l'Imbo au Burundi entre 1890 et 1916 », in Université du Burundi (1985): Questions sur la paysannerie au Burundi, Bujumbura : FLSH,pp. 55-56. 
et 12.000 à Muramba ${ }^{44}$. De 1960 à 1965 , le Congo connut une guerre consécutive aux conditions de son accession à l'indépendance: tentative de sécession de la province minière du Katanga, déstabilisation dans sa partie orientale avec des déplacements de populations congolaises dans la plaine de l'Imbo jusqu'à Nyanza-Lac. Le Congo connaîtra une relative stabilité à partir de 1965 avec la prise du pouvoir par le Maréchal Mobutu. Le Burundi connaîtra de nouveaux des déplacés forcés rwandais entre 1959 et 1964, et en 1973 avant qu'un mouvement inverse ne s'opère suite à la victoire du Front Patriotique Rwandais (FPR) en 1994.

Aussi, les crises récurrentes au Burundi ont également produit des migrations forcées de populations. La période postcoloniale au Burundi aura été marquée par des crises récurrentes produisant des flux de déplacés forcés tant à l'intérieur du pays que dans les pays limitrophes et lointains : déplacés internes dans la région de Muramvya en octobre (centre du Burundi) 1965 suite à une tentative de coup d'Etat contre le Mwami (Roi) Mwambutsa IV Bangiricenge. Particulièrement en avril 1972, le pays connaît une guerre civile (300.000 réfugiés dans les pays limitrophes), en août 1988 (25.000 déplacés internes et réfugiés), la période de la guerre civile entre 1993 et 2000 ont provoqué des vagues de réfugiés pouvant atteindre 600.000 Burundais anciens et nouveaux vivant en Tanzanie, en RD Congo et ailleurs.

Au-delà de cette image peu reluisante, le pays avait à résoudre trois questions fondamentales, à savoir refonder une nouvelle légitimité du pouvoir dans un système républicain, bannir le recours à la violence politique, et enfin sur cette base, s'attaquer aux problèmes collectifs auxquels le pays fait face sur le plan structurel et conjoncturel. Des mouvements de retour au Burundi sont apparus à la suite de différentes circonstances, comme dans les années 1970 et 1980 tandis que d'anciens réfugiés burundais sont massivement rentrés suite aux élections démocratiques de juin 1993 au Burundi, à l'instabilité due à la guerre civile au Rwanda à partir de 1990 et le génocide de 1994, et à l'insécurité en République Démocratique du Congo dans les années 1990. Pendant la période de consolidation de la paix consécutive à l'application de l'Accord d'Arusha pour la paix et la réconciliation au Burundi signé en août 2000, plus de 600.000 anciens et nouveaux réfugiés burundais en Tanzanie et ailleurs ont volontairement regagné le Burundi avec l'assistance du HCR, de la Croix Rouge et des pays directement concernés.

La crise dite du «troisième mandat» d'avril 2015 et les mouvements contestataires qui s'en sont suivis ont conduit des Burundais à chercher refuge principalement dans les pays limitrophes. D'après le HCR, le nombre de réfugiés burundais était estimé à 332.840 au 31 décembre 2019 dont 166.978 en Tanzanie, 72.000 au Rwanda, 47.000 en RD Congo, 45.000 en Ouganda. La quasi-totalité de ces réfugiés avaient quitté le pays il y a près de cinq ans, au lendemain de la crise politique d'avril 2015. Ceci dit, entre janvier et décembre 2019, 21.197 réfugiés ont été rapatriés de la Tanzanie d'après la Direction générale chargée du rapatriement des réfugiés au Ministère de l'Intérieur ${ }^{45}$.

Il s'agit dans ces différents cas de figure de migrations forcées, croisées et subies consécutives à des conflits internes où il faut accueillir et accorder l'asile sur base du principe «prima faciès». A

\footnotetext{
${ }^{44}$ Voir à ce sujet Gahama Joseph, «La question des réfugiés », in Chrétien J-Pierre \& Mukuri Melchior (sous la dir.) (2002), Burundi, la fracture identitaire. Logiques de violence et certitudes «ethniques», Paris, Karthala, pp.225-238.

${ }^{45}$ Voir Boddaert Mathilde, "Réfugiés burundais », http://www.crisisgroup.org visité le 14 novembre 2016 ; le site www.arib.info visité le 14.01.2020.
} 
titre d'exemple, l'Office National pour la Protection des Apatrides (ONPRA) ${ }^{46}$ a enregistré au 31 décembre 2015, 2.931 demandeurs d'asile au Burundi en provenance des pays limitrophes, tandis qu'au 31 octobre 2016, 56.050 réfugiés et 3.347 demandeurs d'asile, 57.926 personnes déplacées internes et 974 personnes à risque d'apatridie. Ils étaient à $99 \%$ originaires de la République démocratique du Congo.

\section{Des déplacements forcés dus aux catastrophes naturelles}

Le Burundi a connu des flux de déplacements de population internes ou interrégionaux fruits des catastrophes naturels : effondrements des berges et inondations consécutives en février 2014 au nord de Bujumbura ou le long du Lac Tanganyika pendant le premier semestre de 2015 et ailleurs. Ces évènements, spectaculaires soient-ils, sont venus rappeler la fragilité du pays face à des hasards naturels et la nécessité de mettre sur pied une politique de lutte contre les catastrophes naturelles. Ces derniers sont de divers ordres: les vents violents, des pluies diluviennes suivies d'inondations, des incendies de bâtiments ou de brousse, des glissements de terrain, des chutes de grêles, des tremblements de terre, des températures excessives, des tonnerres, la foudre, des infections causées par des parasites, des pandémies, etc.

L'Organisation Internationale des Migrations (OIM) en charge de contribuer à une migration internationale ordonnée à travers le monde fait état dans ses rapports mensuels de mouvements de population internes dus aux catastrophes naturelles. Ces dernières sont des pluies torrentielles et des cas de glissements de terrain y relatifs, des vents violents et la sècheresse.

Tableau No2 : Populations internes déplacées entre août et décembre 2019

\begin{tabular}{|l|l|l|l|l|l|}
\hline Mois & Août & Septembre & Octobre & Novembre & Décembre \\
\hline $\begin{array}{l}\text { Personnes } \\
\text { déplacées } \\
\text { internes }\end{array}$ & $\begin{array}{l}106.197 \text { PDI ou } \\
23.752 \text { ménages }\end{array}$ & $\begin{array}{l}103.352 \text { PDI ou } \\
23.180 \text { ménages }\end{array}$ & $\begin{array}{l}103.352 \text { PDI ou } \\
23.162 \text { ménages }\end{array}$ & $\begin{array}{l}102.722 \text { PDI ou } \\
23.009 \text { ménages }\end{array}$ & $\begin{array}{l}104.191 \text { PDI ou } \\
23.343 \text { ménages }\end{array}$ \\
\hline $\begin{array}{l}\text { Motif: } \\
\text { désastres } \\
\text { annuels }\end{array}$ & $77 \% / 23 \%$ & $77 \% / 23 \%$ & $79 \% / 21 \%$ & $79 \% / 21 \%$ & $79 \% / 21$ \\
\hline
\end{tabular}

Source : Auteur sur base des rapports de l'OIM dits Tableau de bord ('Dashboard') des déplacements internes PDI : Personnes Déplacées Internes.

Ces chiffres font preuve de flux de déplacements forcés internes dus en moyenne à $78 \%$ aux catastrophes naturelles et presque tout le pays est affecté à des degrés divers, particulièrement les provinces de l'Ouest et celles du Centre et de l'Est sont particulièrement et régulièrement touchées par ces désastres naturels. Les 22\% restants n’ont pas été clairement identifiés dans les rapports mensuels. Ceci dit, les intentions de retour sont ainsi exprimées pour l'année 2019 : 62\% souhaiteraient un retour dans leur communauté d'origine, 34\% une intégration locale, 3\% une réinstallation ailleurs dans le pays et $1 \%$ une réinstallation dans un autre pays.

${ }^{46}$ ONPRA, HCR \& IGEBU, Rapport du HCR au 31 octobre 2016. 
Tableau $\mathrm{N}^{\circ} 3$ : Effets des catastrophes naturelles du 05 au 11 janvier 2020

\begin{tabular}{|l|c|c|c|}
\hline Types d'urgences & Personnes affectées & Personnes déplacées & Personnes concernées \\
\hline Pluies torrentielles & 152 & 42 & 192 \\
\hline Glissement de terrain & 588 & 588 & 1176 \\
\hline Total & 740 & 630 & 1370 \\
\hline
\end{tabular}

Source : Rapport mensuel de l'OIM

Sur le plan structurel, il existe depuis le 19 avril 2018 un ministère ayant pour attributions, entre autre, la gestion des catastrophes ; une Direction générale chargée de la protection civile et une plateforme nationale de prévention des risques et de gestion des catastrophes. Par réduction des risques de catastrophes, il faut entendre la réduction de l'intensité des catastrophes, l'apport d'une protection et la proposition d'une réponse aux facteurs d'exposition des populations à des catastrophes de toute nature ; c'est enfin de compte protéger des vies humaines et des biens des communautés concernées.

L'Assemblée Nationale du Burundi a ratifié le 31 octobre 2012 un projet de loi portant ratification de la Convention de Tampere (Finlande) dite Traité International sur les télécommunications d'urgence signée dans cette ville le 18 juin 1998. Concrètement, la ratification de ce traité conditionne la mise à la disposition d'un pays par l'Organisation des Nations Unies des moyens de communication d'urgence pendant les opérations de secours en vue d'atténuer les effets négatifs des catastrophes. Ceci dit, des défis existent encore tels que la mise sur pied d'une cartographie et d'une base de donnée des risques de catastrophes, et une législation en la matière; de surcroît un fonds propre de prévention et de gestion des urgences serait également nécessaire ${ }^{47}$.

\section{D'un statut à un autre : Une fuite des cerveaux}

Pendant la Guerre froide, les deux blocs et leurs alliés attiraient étudiants et cadres supérieurs dans leurs pays respectifs. Ils sont de futurs cadres et membres de l'élite de leur pays d'origine, sans doute de futurs interlocuteurs qu'il fallait former et montrer le modèle de civilisation à adopter. Si le départ massif d'étudiants parfois à la fin de leur cycle des humanités dans les pays des deux blocs encore rivaux s'est estompé avec la fin de la guerre froide en 1989, des programmes existent encore. Les étudiants bénéficient aujourd'hui d'un régime souple d'entrée dans le pays de leur choix, des programmes adaptés existent comme Campus France, le Service allemand d'échange académique (Deutsche Akademischen Austausch Dienst, DAAD), le Programme Fullbright (USA), le Programme Erasmus (Union Européenne), etc. Des étudiants burundais finissent aujourd'hui par devenir des migrants de travail, de demandeurs d'asile en fonction de l'évolution de la situation politique dans leur pays d'origine, voire émigrer vers d'autres cieux comme l'Amérique du Nord, l'Australie et ailleurs.

Dans cet ordre d'idées, le Magazine Iwacu nous apprend qu'il existe actuellement en France plus de médecins burundais qu'au Burundi ${ }^{48}$; ils sont partis pour la plupart faire leurs études

\footnotetext{
${ }^{47}$ Burundi Eco du 01 juin 2018.

${ }^{48}$ Magazine Iwacu, $\mathrm{N}^{\circ} 1$, Novembre 2011 : «Où sont nos cerveaux ? ».
} 
supérieures et ne sont plus rentrés dans leur patrie d'origine. En Octobre 2007, sur 340 enseignants attendus, 211 enseignants, soit 65\%, ne se retrouvent pas au Burundi ou sont partis pour le Rwanda où, dans une politique de remplacement des cadres décédés pendant le génocide de 1994, le Programme des Nations Unies pour le Développement (PNUD) proposait des conditions de salaires et de séjour attrayantes ${ }^{49}$. D'après le Professeur Gabriel Ndayisaba, de 1993 à 2007, sur plus de 100 médecins partis se spécialiser en Europe, en France particulièrement, seul $1 / 3$ est rentré au Burundi.

Le programme MIDA-Grands Lacs initié par l'Organisation Internationale des Migrations (OIM) et le gouvernement du Royaume de Belgique et le programme TOKTEN initié par le PNUD n'ont pas pu résoudre entièrement le problème. Les candidats, après avoir mis à contribution leur savoir dans des domaines aussi divers que l'éducation, la santé, l'agriculture ne se sont pas finalement décidés à s'implanter définitivement dans leur pays d'origine. Les raisons sont évidentes à savoir l'insécurité qui régnait dans le pays puisqu'avant 1993 la plupart des enseignants rentraient au pays une fois leurs études terminées; la pauvreté ou les conditions de travail et enfin, on peut ajouter la situation personnelle (volonté, courage, scolarité des enfants, nouvelles perspectives dans le pays d'accueil, etc.) ${ }^{50}$. Il faudrait d'autres enquêtes chiffrées pour évaluer dans quelle proportion des individus, par famille entière, continuent à émigrer vers la Belgique, le Canada, l'Australie et ailleurs.

\section{Communauté omanaise et Apatridie : un imbroglio humain et juridique}

$\mathrm{Au}$ Burundi, des ressortissants d'origine omanaise sont dans une situation d'apatridie pour des raisons historiques et juridiques, ces derniers ou plutôt leurs grands-parents s'étant installés au Burundi depuis la fin du XIX ${ }^{\text {ème }}$ siècle pour faire du commerce ${ }^{51}$. Entre-temps, certains ont vu leurs pièces d'identité perdre leur valeur et leur progéniture n'a plus de documents valables attestant leur nationalité. Parmi ceux qui sont rentrés pour renouveler leurs documents perdus ou invalides, certains sont retournés sans en avoir obtenu car selon la loi omanaise, «ce renouvellement de passeport est conditionné par la présentation d'une preuve de nationalité omanaise du père, validée par les chefs des clans et une demande de récupération de nationalité sans prétention aux droits des autres citoyens omanais» ${ }^{52}$. Rappelons que 1'apatridie constitue plutôt une situation juridique et d'après un ressortissant concerné lors de mes entretiens, la communauté dite omanaise a découvert la gravité de sa situation particulièrement en 1995 quand, suite à une lecture minutieuse de la loi sur les étrangers, le gouvernement de cette époque a refusé de leur renouveler la carte de séjour pour étranger invoquant le motif que ce document est

\footnotetext{
4918 professeurs ont presté dans ce pays dont 5 au Kigali Institute of Education (KIE); Voir Midende Gilbert, Problématique de la fuite des cerveaux à l'université du Burundi, Bujumbura, PNUD, 2007.

${ }^{50}$ Iwacu $\mathrm{N}^{\circ} 1$ de Novembre 2011: Interview de Déo Christian Haringanji : «Pourquoi partent-ils, comment peuventils revenir? pp.6-8 et celle du Prof. Julien Nimubona : «Il faut valoriser la science », p. 29-30.

51 Voir à ce sujet : "Stateless in Burundi : Omanis search for a nationality" ; in http://www.english.alarabiya.net consulté le 04 juillet 2017.

${ }^{52}$ Ntwari Bernard (2013) : Risque d'apatridie au Burundi: Cas des Omanais vivant au Burundi, Mémoire, DESS en Droits de l'Homme et Résolution pacifique des conflits, p. 9.
} 
octroyé en principe à un ressortissant étranger qui possède une nationalité étrangère avec des documents, passeport entre autres, qui le prouvent.

De cet imbroglio à la fois politique, juridique et social, il en résulte des statistiques consolidées après une opération d'enregistrement des personnes d'origine omanaise menée par l'Office National pour les Réfugiés et Apatrides (ONPRA) avec l'appui du HCR du 30 mai au 08 juin 2012 que le nombre de ressortissants omanais enregistrés s'élève à 1.302 personnes, dont 622 femmes et 680 hommes comme vivant au Burundi et 590 personnes comme directement «non concernées». Il s'agit pour ces derniers des conjoints et des enfants des ressortissants omanais ayant une autre nationalité burundaise, rwandaise, congolaise, indienne, pakistanaise et autres. Les négociations tripartites entre le Gouvernement du Burundi, le Haut-Commissariat des Nations Unies pour les réfugiés et les représentants de la communauté omanaise avaient conclu aux quatre éventuelles voies de sortie suivantes :

(1) L'octroi de la nationalité omanaise à la suite d'une démarche du gouvernement du Burundi auprès du Sultanat d'Oman. La procédure se réalise dans le Sultanat d'Oman, un Etat de 3.000 .000 d'habitants dont $40 \%$ sont des étrangers et qui enregistre chaque année 500.000 demandes de passeport ;

(2) La naturalisation, ce qui contribuerait à vider la question en octroyant la nationalité burundaise à ces ressortissants qui sont pour la plupart intégrés depuis longtemps au Burundi, certains exerçant même des professions de haut niveau;

(3) La réinstallation dans d'autres pays qui le font également pour les réfugiés comme les EtatsUnis d'Amérique, le Canada, la Norvège, l'Australie et autres. Cette solution s'avère difficile à mettre en œuvre car la communauté omanaise n'a en réalité pas de véritable statut juridique, ce qui pourrait être réalisé par le HCR et l'OIM en envisageant la solution suivante; et enfin

(4) L'octroi du statut d'apatride : C'est une situation qu'aucun des partenaires ne veut pas en réalité, à savoir les pays concernés- le Gouvernement du Burundi, le Sultanat d'Oman-, le HCR et la communauté omanaise concernée au premier chef car ce statut les empêcherait de facto sans doute à jamais de regagner la terre de leurs ancêtres ${ }^{53}$. Pourtant, d'après mes interlocuteurs, cette solution envisagée obligerait d'un côté le pays hôte à leur assurer des mesures de protection juridique, et de l'autre, elle permettrait au HCR d'envisager une éventuelle réinstallation dans un pays tiers.

Quelle que soit leur appellation, «personnes sans statut légal», «personnes à risque d'apatridie» ou «personnes sans droits», ces ressortissants omanais et leurs proches sont confrontés à plusieurs défis tels que le manque de documents valables, et donc l'impossibilité d'enregistrer les naissances et obtenir des actes de naissance puisque la loi burundaise n'autorise pas un tel enregistrement en cas de parents sans documents valables attestant de leur nationalité. La majorité parle la langue nationale, le Kirundi, en plus de l'Arabe, certains d'entre eux ont fait des études et donc parlent français, anglais; on compte parmi eux des juristes, des professeurs et des médecins; d'autres exercent divers métiers dans des centres commerciaux tels que la mécanique automobile, le commerce général, le courtage, etc.

\footnotetext{
${ }^{53}$ Ajoutons qu'on peut trouver dans une seule et même famille à la fois des membres apatrides et d'autres ayant la nationalité omanaise, passeport, séjour alternatif dans les deux pays, etc. Voir également «Statelessness in Burundi »; in: http://www.iwacu-burundi.org/english consulté le 04 juillet 2017.
} 
Sur le continent africain, une tentative «éliminer l'apatridie» est en cours. Les pays membres de la Communauté économique des Etats d'Afrique de l'Ouest (Cédéao) qui compte un million d'apatrides (autant en Afrique australe et des dizaines de milliers en Afrique de l'Est) ont adopté le 09 mai 2017 un «plan d'actions régional pour l'éradication de l'apatridie dans l'espace communautaire»d'ici à 2024 lors d'une conférence ministérielle à Banjul (Gambie) ${ }^{54}$. Les mesures concrètes adoptées en vue de «tarir les sources de l'apatridie » se déclinent sur deux piliers à savoir le respect des cadres juridiques, politiques et institutionnels qui régissent l'éradication de l'apatridie, intégrer les conventions internationales portant sur l'apatridie dans leur législation, notamment la Convention sur la réduction de l'apatridie de 1961.

\section{Déplacements forcés et tensions diplomatiques régionales}

La présence de réfugiés conduit naturellement à fixer davantage, sinon à jamais, le conflit interne dans leur pays d'origine, lequel ne peut en aucun cas nier la persistance d'un malaise politique ou économique à l'origine de la fuite de ces nationaux d'un pays. Cette situation se complique davantage en cas de mouvements croisés de réfugiés au niveau régional. Les réfugiés du Rwanda et du Burundi sont remarquables par leur appartenance «ethnique», Tutsi rwandais fuyant la «Révolution rwandaise de 1959» entre 1959 et 1963 et Hutu burundais fuyant le Burundi en 1972-1973 dans les pays limitrophes ont constitué, au-delà de la tragédie humaine et personnelle, des vecteurs de communication tant dans le pays hôte que le pays d'origine. L'impact de la violence et des flux de réfugiés aura été de rigidifier les préjugés ethniques: d'une part, pour les auteurs qui veulent expliquer et justifier leurs actes de violence, ils atteindront leurs objectifs en déshumanisant l'autre; et d'autre part, les différents actes de violence deviennent une partie traumatisante de «l'identité Hutu et Tutsi» confondus, créant dans les deux cas une image de soi de faiblesse et de vulnérabilité ${ }^{55}$.

Depuis avril 2015, date de la candidature de l'actuel Président de la République pour un autre mandat et la crise politique qui s'en est suivie, les relations entre le Burundi et le Rwanda d'un côté sont tendues, le premier accusant le second d'abriter des camps d'entraînement pour réfugiés, donc une source de déstabilisation; la Belgique et d'autres pays européens comme la France d'autre part sont accusées de chercher à déstabiliser le régime en place à Bujumbura ${ }^{56}$. Cette crise des relations entre Etats conduit à un refroidissement diplomatique.

\section{Les limites d'une politique migratoire}

Une politique d'asile consiste à déterminer la base sur laquelle il faut accueillir des migrants, leur accorder l'asile et assurer une protection internationale. Cette politique se traduit par des lois

\footnotetext{
${ }^{54}$ Voir Le Monde du 11 mai 2017 et www.rfi.fr consulté le 10 mai 2017. Pour de plus amples informations sur l'enjeu de l'apatridie et les solutions envisageables, consulter UNHCR \& UIP (2005) : Nationalité et Apatridie. Un guide pour les parlementaires, Genève

${ }_{55}$ Uvin Peter (1998) : Aiding violence. The development enterprise in Rwanda, Connecticut: Kumarian Press, p. 34; voir Willame J-Claude (1995): Aux sources de l'hécatombe rwandaise, Bruxelles, Cedaf.

${ }^{56}$ Selon le gouvernement burundais, les attaques de Kabarore en juillet 2015 et la récente attaque du 17 novembre 2019 contre une position de l'armée régulière ont été menées par de groupes rebelles venus du Rwanda où se trouvent une importante communauté de réfugiés burundais depuis 2015, et ces deux évènements font l'objet de tensions diplomatiques entre les deux pays.
} 
favorables à l'accueil des réfugiés ou restrictives pouvant conduire à l'expulsion des demandeurs reboutés. En vue de sensibiliser le monde et attirer l'attention des gouvernements sur l'ampleur et l'acuité des flux migratoires et les problèmes posés par les réfugiés, le HCR organise chaque année deux événements importants, à savoir la célébration de la Journée mondiale des refugiés et l'attribution de la distinction Nansen. La Journée mondiale des refugiés est commémorée le 20 juin de chaque année à travers le monde et offre l'opportunité aux Etats et gouvernements du monde entier de discuter, d'évaluer la situation des réfugiés dans leurs pays respectifs et dresser le bilan réalisé de leur politique en matière d'accueil et de protection des réfugiés.

Quant au Prix Nansen, nous devons ce nom au célèbre explorateur norvégien des régions polaires, Fridtjof Nansen (1860-1930), également le premier Haut-Commissaire de la Société des Nations (ancêtre de l'Organisation des Nations Unies) en 1921 et qui s'est illustré, en tant que Délégué de la Norvège auprès de ladite institution, dans le rapatriement d'un demi-million de prisonniers de guerre Allemands, Autrichiens et Hongrois. Depuis 1955, est décerné chaque année le Prix Nansen ${ }^{57}$ à des personnalités ou groupes ayant rendu des services aux réfugiés ou contribué à l'œuvre du HCR à travers le monde. Les lauréats présentent des profils aussi divers que Mme Eleanor Roosevelt (1954), épouse du Président américain du même nom, Médecins Sans Frontières (1993), Gracia Machel, épouse en deuxième noce de Nelson Mandela (1995), Marguerite Barankitse (2005), UNMACC \& 1000 démineurs libanais (2008), Aqeela Asifi, une enseignante au Pakistan (2015).

La Déclaration universelle des droits de l'Homme, en son article 14 relatif au droit d'asile, prescrit que «devant la persécution, toute personne a le droit de chercher asile et de bénéficier de l'asile en d'autres pays». Ainsi, ne prescrit-il pas le devoir des Etats de l'accorder, ces derniers ne voulant pas ouvrir leurs frontières à des personnes pouvant constituer pour eux des menaces à la sécurité nationale ${ }^{58}$.

Sur le plan politique, les Etats restent libres en la matière de déterminer leur politique migratoire en général, et leur système national d'asile et de protection des migrants en particulier, et ce dans le respect des conventions auxquelles ils ont souscrit.

En plus des réfugiés ayant décidé de rentrer au Burundi, d'autres ont été réinstallés aux EtatsUnis (13.000 réfugiés entre 2006 et 2007), en Australie, en Norvège, au Canada, en NouvelleZélande, en Belgique tandis que 180.000 réfugiés ont acquis la nationalité tanzanienne et devraient être répartis sur l'ensemble du territoire tanzanien («autres solutions durables») ; en 2015, il restait encore 26.000 réfugiés burundais autour de Kigoma ${ }^{59}$.

Une politique migratoire s'avère difficile à mettre en œuvre dans la mesure où les pays entrent dans plusieurs catégories à la fois en fonction des objectifs réels ou supposés des migrants, un pays pouvant être à la fois une terre de départ, de transit, ou d'accueil. Au sortir de l'Apartheid, l'Afrique du Sud a accueilli des migrants venus de pays africains, coïncidant avec le verrouillage opéré au début des années 1990 par les pays développés, européens et américains en matière d'octroi de visa et des conditions de séjour. La réaction du gouvernement a été la mise sur pied

\footnotetext{
${ }^{57}$ Attribution d'une médaille à l'effigie de Nansen et d'une enveloppe de 100.000\$US.

${ }^{58}$ Fombeno Tagum H.J., op. cit, p.250.

${ }^{59}$ Rapport HCR 2010 tenant compte de ce mouvement consécutif à l'application de l'Accord d'Arusha. D'autres vont s'y ajouter à partir de 2015, nous y reviendrons plus tard.
} 
d'une politique restrictive en matière de séjour des étrangers et la reconduite aux frontières de milliers de clandestins. Une stabilité politique et des perspectives économiques dans son propre pays s'avèrent ainsi la meilleure arme contre le flux de déplacements forcés sinon à titre personnel ou en groupe, l'individu cherchera ailleurs où son épanouissement sera garanti. En dehors de toute considération politique, il se remarque déjà depuis les années 2010 une nouvelle forme de migration vers le sud de l'Ouganda, les pays de l'Afrique australe, l'Asie, la Chine et dans les pays du Moyen Orient (Qatar, Dubaï, Oman) principalement. La diaspora burundaise serait actuellement estimée à 365.000 personnes éparpillées principalement dans les pays suivants: 26.000 en Tanzanie, 100.000 en Ouganda, 20.000 au Kenya, 14.000 aux USA, 6.000 au Canada, 2.000 en Asie et en Océanie ; bref 38 Burundais sur 1000 vivent à l'extérieur du pays ${ }^{60}$. Pour d'autres pays africains, il faudra penser la politique migratoire vu que les migrations interafricaines s'avèrent également importantes comme les statistiques suivantes en font montre. A ce sujet, selon Catherine Withol de Wenden, il faudra relativiser l'image de migrants du Sud venus envahir le Nord; en 2011, les Nations Unies ont répertorié 248 millions de migrants dont seulement 97 millions de personnes nées dans le Sud vivant aujourd'hui dans un pays développé. Cette migration se rapproche de peu des migrations Sud-Sud (74 millions), Nord-Nord (37 millions) et Nord-Sud (40 millions) ${ }^{61}$.

\section{Se conformer aux principes internationaux en la matière}

La protection des demandeurs d'asile et réfugiés est de la responsabilité des Etats en premier lieu. Par conséquent, la législation nationale sur les demandeurs d'asile et réfugiés fondée sur les standards internationaux s'avère indispensable dans la protection de ces derniers et constitue une base juridique à la recherche de solutions durables aux problèmes posés par la présence des réfugiés. L'intégration du droit international dans la législation interne s'avère primordiale dans l'application de la Convention de 1951 et celle de 1967 ainsi que les matières que ces deux textes ne couvrent pas, à savoir les procédures de détermination du statut de réfugié. Il sied donc de revoir les textes applicables en matière de protection des demandeurs d'asile, des réfugiés et des étrangers en général.

Depuis l'année 2015, le gouvernement a commencé à penser une politique des migrations et une autre de la diaspora. Ceci dit, il sied d'analyser ces dernières à travers le cadre juridique régissant la matière, lequel consiste à se conformer à la législation internationale en la matière. Le législateur burundais se réserve une marge de manœuvre dans deux domaines sensibles, à savoir d'une part, la liste des clauses de cessation du statut de réfugié en droit burundais qui dépasse celle prévue par la Convention de 1951 du HCR et la Convention de l'OUA de 1967 qui la voulait limitative et exhaustive. Et d'autre part, elle ne reconnaît pas explicitement les droits reconnus au réfugié tels que le droit à la propriété intellectuelle et industrielle, la liberté de

\footnotetext{
${ }^{60}$ République du Burundi, Politique nationale de la diaspora, Bujumbura, MREC, 2015, pp. 12-15.

${ }^{61}$ Voir Wihtol de Wenden Cathérine : Les nouvelles migrations. Lieux, hommes, politiques, Paris, Ellipses (tiré de http://www.cairn.info visité le 03 .01.2016).
} 
religion, l'acquisition des biens mobiliers et immobiliers qui ne sont pas explicitement reconnus ${ }^{62}$.

\section{Conclusion}

Malgré les progrès que connaît actuellement le monde dans des domaines aussi divers que les médias et la circulation de l'information, l'informatisation et les nouvelles technologies de l'information et de la communication, d'autres fronts restent ouverts. Nous nous sommes intéressé dans cet article à une analyse d'un groupe qui, une fois réuni, pourrait constituer la $21^{\text {ème }}$ nation au monde: 65,3 millions de personnes déplacées en 2015, elles étaient 59,5 millions l'année précédente, tandis que l'ensemble de migrants internationaux s'élève à 250 millions. Les migrants ont envoyé en 2016, 601 milliards de dollars à leurs familles d'origine ; ils ont atteint 70,8 millions en 2018. Ces dimensions macro-économiques et démographiques font état certes de l'ampleur du phénomène, mais elle contribue plutôt à s'interroger sur les causes profondes de ces migrations forcées ou non. La création du Haut-Commissariat des Nations Unies pour les Réfugiés en 1951 avait pour objectif, entre autres, d'accueillir des demandeurs d'asile venant de l'Europe de l'Est. Cette dimension de protection des droits humains pour des hommes et des femmes cherchant la liberté à l'Ouest ne pouvait pas à terme ignorer d'autres causes de déplacements forcés à travers le monde: conflits, guerres, religion, catastrophes naturelles, politique, recherche de meilleures opportunités économiques et sociales ailleurs et flux migratoires qui en sont la conséquence naturelle obligent les Nations unies à redéfinir le concept original de réfugié en vue de s'appliquer à de nouveaux cas de figure dans le monde ${ }^{63}$.

Si ces déplacements forcés ne remettent pas en cause l'équilibre du monde, il n'en reste pas moins qu'ils affectent durablement les pays, régions et populations concernés. Le rôle de la présence des réfugiés est, entre autres, de fixer à terme le problème, soit-il politique, économique et autre à cause duquel un individu a opté pour le chemin de l'exil. Ces réfugiés restent pour la majorité d'entre eux dans la région d'origine, alimentant davantage les conflits dans des pays qui sont déjà suffisamment accablés par l'enjeu de sortie du sous-développement.

Le Burundi comme les autres pays de la Région des Grands Lacs accueille sur son sol toutes les catégories de migrants forcés : demandeurs d'asile, réfugiés, apatrides, originaires à $99 \%$ de la Région, et des personnes déplacées internes tandis que des Burundais vivent dans les pays limitrophes et ailleurs. Le problème posé par la présence des réfugiés, des personnes déplacées et des apatrides ne peut être résolu qu'en affrontant le mal à la racine, à savoir l'origine supposée du problème. Il est d'abord d'ordre politique, se traduisant en conflits de pouvoir qui se règlent, dans certains cas, par la violence ou l'éclatement de guerres civiles avec pour conséquence, entre autres, la fuite de milliers de personnes dans les pays limitrophes et au-delà. Il est également d'ordre économique dans la mesure où en dehors de la situation politique, des individus continuent à chercher un mieux-être ailleurs. Ainsi, une fois la paix retrouvée, les Etats consolidés devraient s'occuper à renforcer davantage le système politique choisi, un système

\footnotetext{
${ }^{62}$ Manirakiza Egide, op. cit, pp.15-16.

${ }^{63}$ Reconduit tous les cinq ans par une Résolution de l'Assemblée Générale des Nations Unies, le mandat du HCR est devenu illimité depuis 2004 «jusqu'à ce que la question des réfugiés dans le monde soit définitivement résolue».
} 
démocratique et libéral, construire la nation, et surtout mettre sur pied une économie fonctionnelle.

\section{Références bibliographiques}

Banque Mondiale (2019) : Leveraging economic Migration for development. A Briefing for the World Bank Board, Washington DC : World Bank Group

Bendl Regine, Hanappi-Egger Edeltraud \& Hofmann Roswitha (2012) : Diversität und Diversitäts- management, Wien : Facukltas Verlag AG.

Chetail Vincent (sous la dir.) (2007) : Mondialisation, migration et droits de l'homme: Le droit international en question, Bruxelles : Bruylant SA.

Chrétien Jean-Pierre \& Mukuri Mukuri (sous la dir.) (2002) : Burundi, la fracture identitaire. Logiques de violence et certitudes «ethniques », Paris : Karthala.

Collins Alan (2013): Contemporary security studies, Oxford: Oxford University Press.

Convention de Genève relative aux réfugiés du 28 juillet 1951.

Déclaration Universelle des droits de l'Homme de 1948.

De Montclos Marc-Pérouse (1998) : «Les flux des réfugiés: un enjeu politique au Sud comme au Nord », La Chronique du CEPED, ³1, Octobre-Décembre 1998, pp.1-4.

Drain Michel \& Dubernet Cécile (2018) : Relations internationales, $23^{\text {ème }}$ édition. Bruxelles : Edition Bruylant.

Garner-Moyer Hélène (2006) : «Gestion de la diversité et enjeux de la gestion des ressources humaines »; in Management et Avenir, 2006/1 ( $\left.{ }^{\circ} 7\right)$, pp. 23-42; DOI: 10.3917/mav.007.0023.

Jaffrelot Christophe \& Lequesne Christian (sous la dir.) (2009) : L'enjeu mondial. Les migrations, Paris : Presses de Sciences Po-L'Express.

Kirsch Olivier (2015) : Migrations internationales: Un enjeu planétaire, Paris : Ed. des journaux officiels

KNOMAD (2016) : Recueil des statistiques sur les migrations et les envois de fonds, Washington : Banque mondiale ; www.knomad.org.

Manirakiza Egide (2014) : Cadre juridique de protection des réfugiés au Burundi. Thème présenté à l'occasion de la Journée Mondiale du Réfugié, 17 juin 2014, Bujumbura: Avocats sans Frontières (ASF).

Manirakiza Egide (2014) : Etude ASF dans le cadre du projet PIDDAR pour avoir une vue globale de la situation juridique des demandeurs d'asile au Burundi, des lacunes de la loi et des difficultés en matière de protection effective des droits pour les demandeurs d'asile, Bujumbura : Avocats Sans Frontières (ASF).

National Intelligence Council (2008) : « Democratization in Africa : What Progress toward Institutionalization? » Conference Report, February 2009.

Ntwari Bernard (2013) : Risque d'apatridie au Burundi : Cas des Omanais vivant au Burundi, Université du Burundi, Mémoire de DESS en Droits de l'Homme et Résolution pacifique des Conflits, Bujumbura.

Organisation Internationale des Migrations (2015) : Etat de la migration dans le monde. Les migrants et les villes : de nouveaux partenariats pour gérer la mobilité, Genève.

Oudjani Jean-Marie (2009) : Éléments constitutifs de l'Etat, Paris, inédit.

Queloz Nicolas \& Bütikofer-Repond Frédérique et alii, (2005): Délinquance des jeunes et justice 
des mineurs. Les défis des migrations et de la pluralité ethnique, Bruxelles : Bruylant SA. République du Burundi (2016) : Politique nationale de la Diaspora, Bujumbura : MREC. République du Burundi (2016) : Politique nationale de la Migration, Bujumbura : MREC. Rolland Cécilia (2013) : Decreased Polarization through economic integration ? A study of ethnic polarization of membres of Rwandes diaspora 19 years after the genocide, Master thesis in Peace and Conflict Studies, Uppsala University : DPCR.

UNHCR \& UIP (2005) : Nationalité et Apatridie. Un guide pour les Parlementaires, Genève.

Université du Burundi (1985) : Questions sur la paysannerie au Burundi, Actes de la Table ronde organisée par la Faculté des lettres et sciences humaines, Bujumbura du 07 au 11 mai 1985.

Uvin Peter (1998): Aiding violence. The humanitarian enterprise in Rwanda, Connecticut: Kumarian press.

Uvin Peter (1999): «Ethnicity and power in Burundi and Rwanda: Different paths to mass violence», Comparative Politics, Vol. 31, N³, April 1999, pp.253-271.

Wihtol de Wenden Catherine (2013) : Les nouvelles migrations. Lieux, hommes, politiques, Paris : Ellipses.

Willame Jean-Claude (1995) : Aux sources de l'hécatombe rwandaise, Bruxelles : CEDAF.

Wondwosen Teshome (2009) : "Opposition Parties in the Politics of Opposition in Africa : A Critical Analysis »; In International Journal of Human and Social Sciences 4 :5, pp. 287301. 